\title{
A numerical and experimental investigation of wave generated by submerged landslides
}

\author{
A Thesis \\ Submitted to the Faculty of Graduate Studies and Research \\ In Partial Fulfillment of the Requirements \\ For the Degree of \\ Master of Applied Science \\ in \\ Environmental System Engineering \\ University of Regina \\ By \\ Haojia Li \\ Regina, Saskatchewan \\ July, 2019 \\ Copyright 2019: H.Li
}




\section{UNIVERSITY OF REGINA}

\section{FACULTY OF GRADUATE STUDIES AND RESEARCH SUPERVISORY AND EXAMINING COMMITTEE}

Haojia Li, candidate for the degree of Master of Applied Science in Environmental Systems Engineering, has presented a thesis titled, A Numerical and Experimental Investigation of Wave Generated by Submerged Landslides, in an oral examination held on July 25, 2019. The following committee members have found the thesis acceptable in form and content, and that the candidate demonstrated satisfactory knowledge of the subject material.

External Examiner: Dr. Rene Mayorga, Industrial Systems Engineering

Supervisor: $\quad$ Dr. Yee-Chung Jin, Environmental Systems Engineering

Committee Member: $\quad$ Dr. Peng Wu, Environmental Systems Engineering

Committee Member: $\quad$ Dr. Amgad Salama, Environmental Systems Engineering

Chair of Defense: Dr. Lisa Fan, Department of Computer Science 


\begin{abstract}
The Weakly-Compressible Moving Particle Semi-implicit (WC-MPS) approach is applied to investigate the wave propagation and the flow fields from sliding blocks. Two bed inclined slope angles at $40^{\circ}$ and $50^{\circ}$ are used to obtain the experimental results. Existing studies have focused on an inclination slope of $45^{\circ}$. The characteristics of the free surface at the two specific angles of $40^{\circ}$ and $50^{\circ}$ have yet to be examined in detail, where $50^{\circ}$ is the maximum angle in the experimentation. Sliding blocks are released under water and the top edges are parallel to the water surface. The simulation results are compared to the experimental measurements on the moving water surface and the velocity distribution at specific locations. To analyze the flow fields, contour and vector plots illustrate the flow characteristics for the entire velocity distribution in the simulation domain along with the vortex movements from the sliding block. Two case scenarios are presented to investigate the velocity distribution of the sliding block. For the first case scenario, seven crosssections, including the front edge, the top edge, and the back edge of the sliding block were selected to further identify the characteristics of the horizontal and vertical velocity distributions. The second case scenario has five specific locations at the front, top and back edge of the block. A good agreement can be observed between the WC-MPS simulated results to the experimental data.
\end{abstract}




\section{Acknowledgement}

I would like to express my sincerest appreciation to my supervisor, Professor Yee-Chung Jin, for his mentorship, guidance, and encouragement throughout all stages of this study.

I would also like to express my gratitude to the members of my thesis committee, Dr.Peng Wu, Dr.Amgad Salama and Dr. Rene Mayorga for reviewing this thesis and sharing their constructive comments.

I acknowledge the financial support of Faculty of Graduate Studies and Research, at the University of Regina and the Natural Science and Engineering Research Council of Canada. 


\section{Table of Contents}

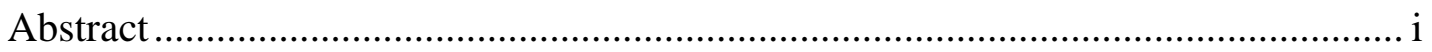

Acknowledgement .......................................................................................... ii

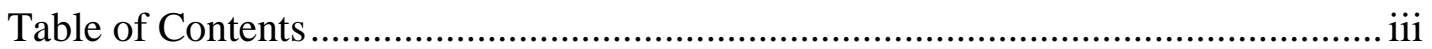

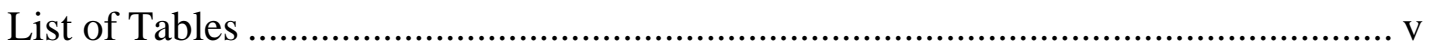

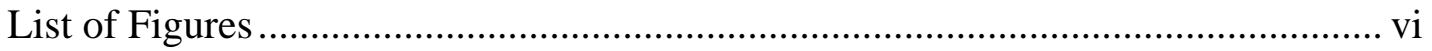

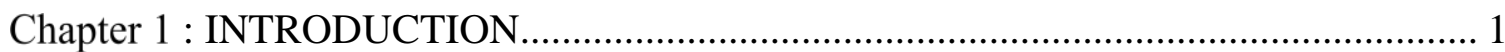

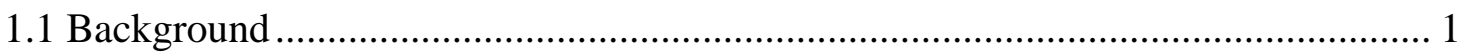

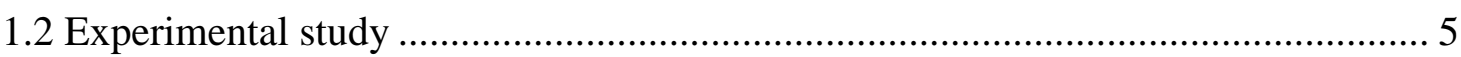

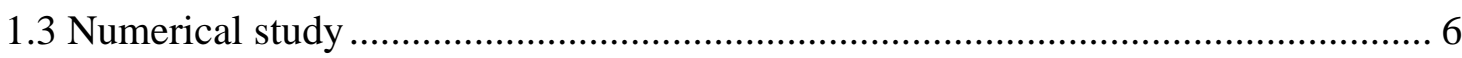

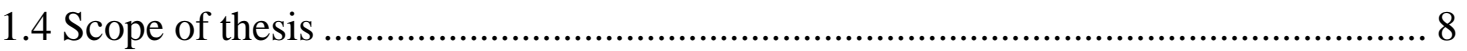

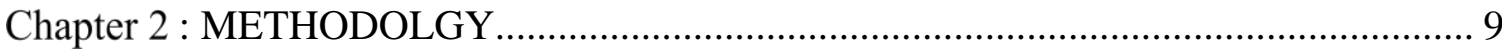

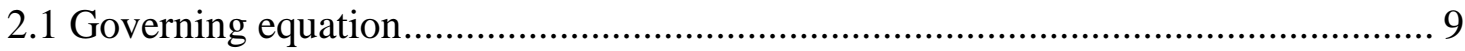

2.2 Weighting function ................................................................................. 10

2.3 Neighbour search strategy...................................................................... 12

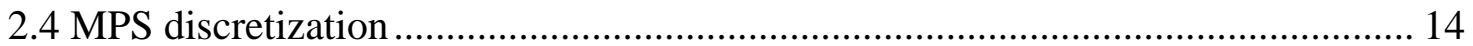

2.5 Equation of State for pressure calculation ...................................................... 16

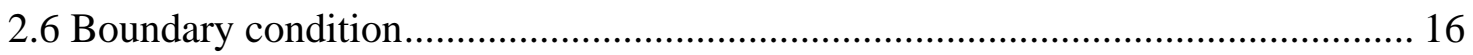

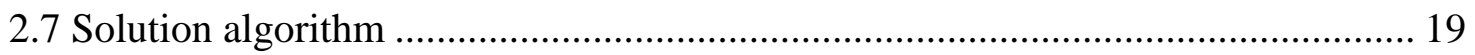

Chapter 3 : EXPERIMENTS SET-UP............................................................... 24 
4.1 A validation of water-surface....................................................................... 28

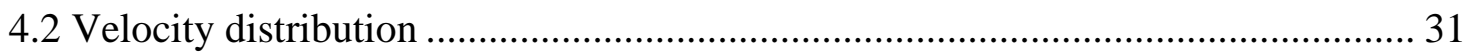

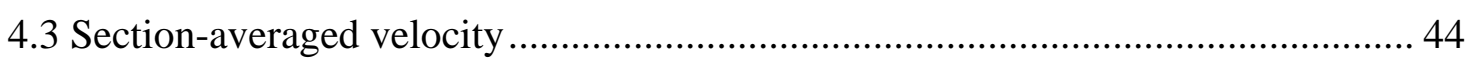

4.4 Velocity contour and Vorticity …………….................................................... 46

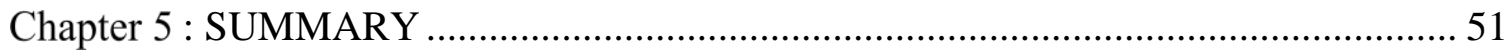

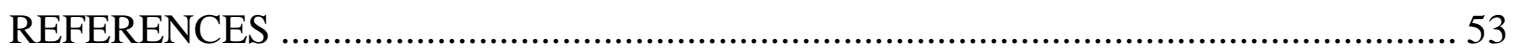

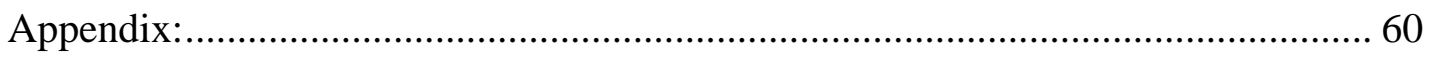




\section{List of Tables}

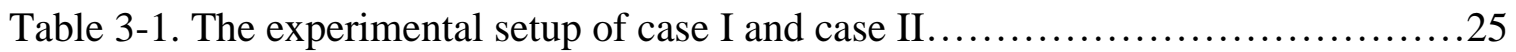




\section{List of Figures}

Figure 1-1. Shows the dimensions of the landslides of Lituya Bay case (Photos: courtesy

of Charles L. Mader)............................................................................................. 3

Figure 1-2. Shows the town of Longarone before and after the Vainot case occurred. (1963

Vajont Survivor's Committee Image source) …......................................................... 4

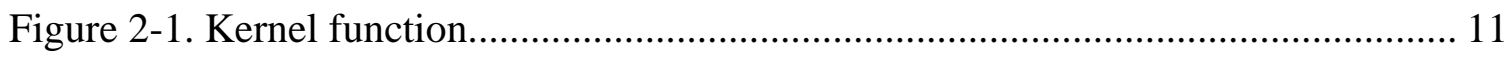

Figure 2-2. Sketch of searching neighboring particles algorithm............................... 14

Figure 2-3. The solid boundary and Ghost particles ................................................ 18

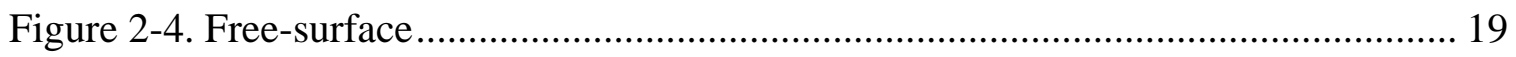

Figure 2-5. Flowchart of Computational algorithm................................................ 23

Figure 3-1. Snapshots during the experiments. (a) case I, where the inclined slope angle is

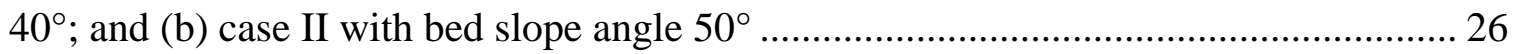

Figure 3-2. (a)The initial condition of experimental set-up. (b) Two different block types.

Figure 4-1. Case I: the water surface comparison between simulation result and

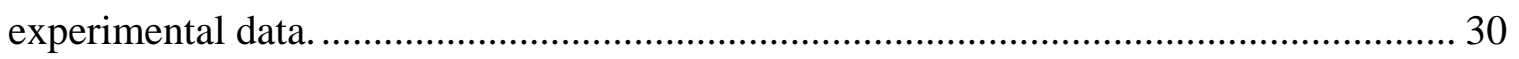

Figure 4-2. Case II: the water surface comparison between simulation result and

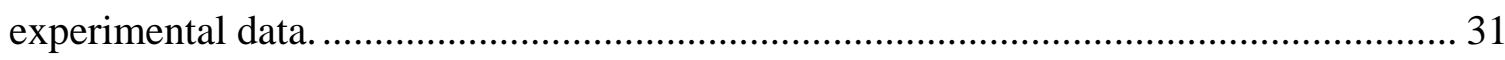

Figure 4-3. Comparison of velocity distribution at the top corner of the slider (cross-section

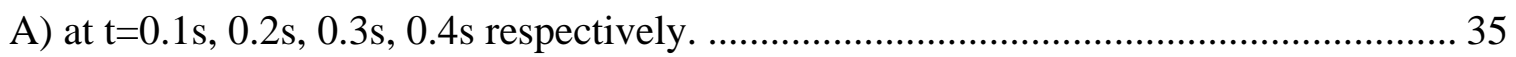

Figure 4-4. The comparison of velocity distribution at the cross-section $G$ and $F$. The comparison is available for cross-section $\mathrm{F}$ at $t=0.1 \mathrm{~s}, 0.2 \mathrm{~s}, 0.3 \mathrm{~s}$ and $\mathrm{G}$ at $t=0.1 \mathrm{~s}$ and $0.2 \mathrm{~s}$. 
Figure 4-5. The comparison of velocity distribution for the cross-section D and $\mathrm{E}$ at $t=0.3 \mathrm{~s}$ and $0.4 \mathrm{~s}$. 37

Figure 4-6. The comparison of velocity distribution for the cross-section $\mathrm{C}$ and $\mathrm{B}$ at $t=0.3 \mathrm{~s}$ and $0.4 \mathrm{~s}$. 38

Figure 4-7. The comparison of velocity distribution for the cross-section A and G of Case

II at $t=0.1 \mathrm{~s}, 0.2 \mathrm{~s}$, and $0.3 \mathrm{~s}$. 42

Figure 4-8. The comparison of velocity distribution for the cross-section C, D and E of

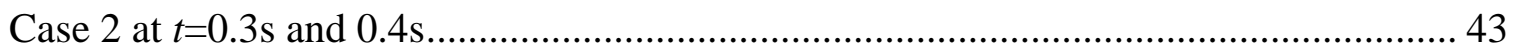

Figure 4-9. Section-averaged velocity distribution of Case I and Case II ...................... 45

Figure 4-10. Velocity contour of case I (left) and case II (right) ................................. 49

Figure 4-11. Vorticity with the vector plot; left part is showing Case I, and right side are

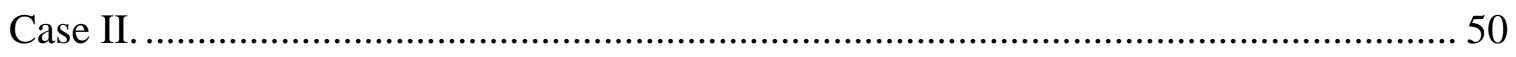

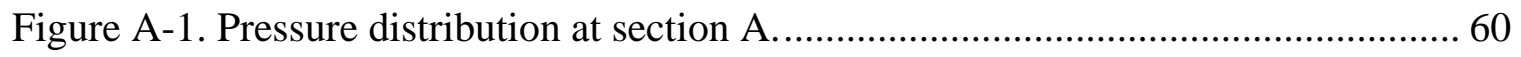

Figure A-2. Pressure distribution at section C.................................................... 61

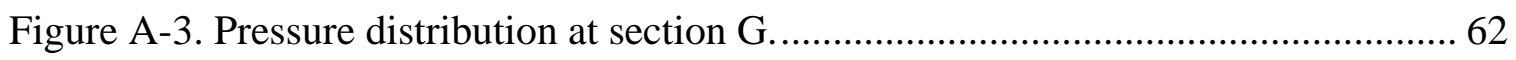

Figure A-4. Pressure distribution from section $\mathrm{C}$ to section A horizontally. ................... 63

Figure A-5. The comparison of velocity distribution between the experimental data and simulated results with different particle size $(0.002 \mathrm{~m}$ and $0.005 \mathrm{~m})$ at section A in Case I 


\section{Chapter 1 : INTRODUCTION}

\subsection{Background}

Wave generated by landslide occurs when a large mass moving down the slope disturb the overlying water and create tsunami waves. Based on the initial condition of the landslide masses and water body interaction, there are three commonly classifications used to identify the categories of the landslide: submarine, subaerial, and partially submerged. Generally, the tsunamis (impulsive waves) are occurred in most of the water bodies including the reservoirs, mountainous rivers, lakes, and oceans (Fritz, 2002). Due to the speed of tsunami wave propagation, the landslide-tsunami hazard has become destructive natural disasters threatening life and infrastructure. As showing in Figure 1-1, on July 8, 1958, a rockslide generated wave ran up to $524 \mathrm{~m}$ causing an approximate $10 \mathrm{~km}^{2}$ destruction areas at the Lituya Bay (Fritz et al., 2001). Moreover, another famous engineering disaster is known as the 1963 Vajont case in Italy (Müller-Salzburg, 1987), which occurred by about $700,000 \mathrm{~m}^{3}$ of sliding masses causing a $260 \mathrm{~m}$ height of the wave above lake level (Figure 1-2). While, over 2000 people has been washed away in villages and towns since the flood descended the Piave River Valley (Müller-Salzburg, 1987). Slingerland and Voight (1979) summarized many worldwide cases of the generated landslides-tsunami. The impact of the subaerial landslide is devastating; another recent example in 2015, a large landslide with 180 million tons of rock was sent into Taan Fiord, Alaska, the glacial retreat, contributed an unstable slope and generated tsunami resulting in a wave reach to $193 \mathrm{~m}$ high. As a result, one of the highest tsunamis scale was detected 
(Higman et al., 2018). These reviews provide the importance of investigating on the occurrences and properties of the tsunami wave.

The study of the physical properties of the generated landslide-tsunamis (impulsive waves) has a dramatic increasing in recent decades. Many researchers are focusing on the configuration of the impulsive waves including the amplitude of the wave (Heinrich, 1992; Wiegel, 1955). Meanwhile, the affecting factors of the impulsive waves have attracted the researchers to pay more attention. In order to understand the complexities of the impulsive waves, investigations of the landslides on different affecting factors namely water depth, velocity of sliding masses, configuration of sliding masses, initial condition of landslides, and inclined slope angle have been studied by several researchers (Ataie-Ashtiani and NikKhah, 2008; Ataie-Ashtiani and Shobeyri, 2008; Fritz, 2002; Grilli and Watts, 2005; Heller et al., 2016; Jin et al., 2016; Watts, 1997). Despite the investigation of these affecting factors, the inclined slope angle indicates a significant role related to the real life since the gravitational force applied the major external force into these specific landslide cases. Thus, researchers start to investigate the effectiveness by using the experimental test, and numerical simulation. 


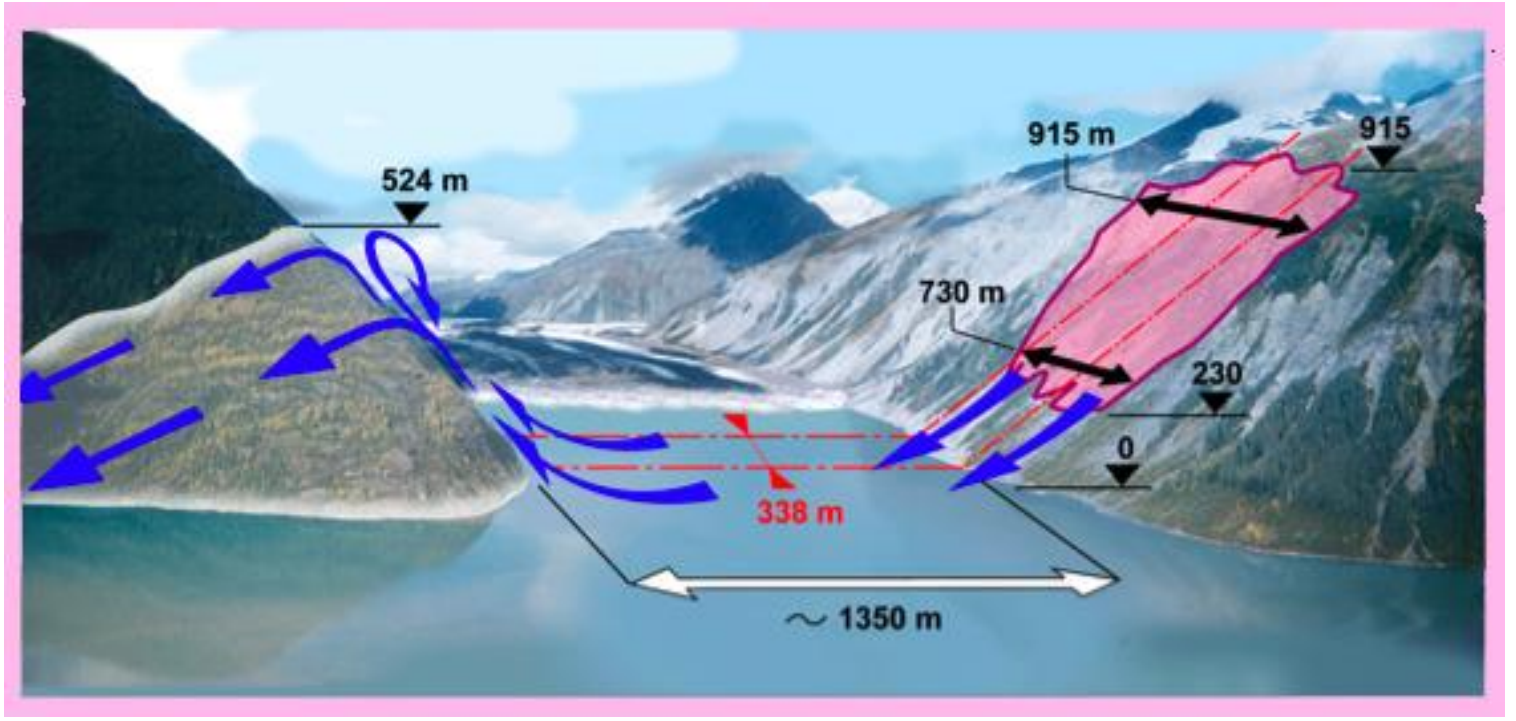

Figure 1-1. Shows the dimensions of the landslides of Lituya Bay case (Photos: courtesy of Charles L. Mader). 


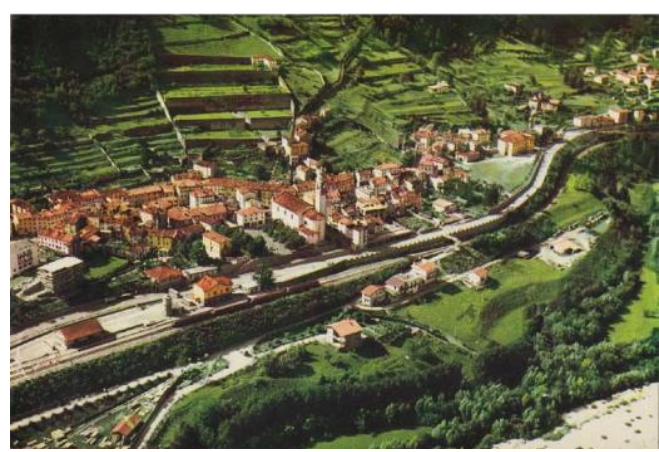

The town of Longarone, Italy, before the landslide, 1963.

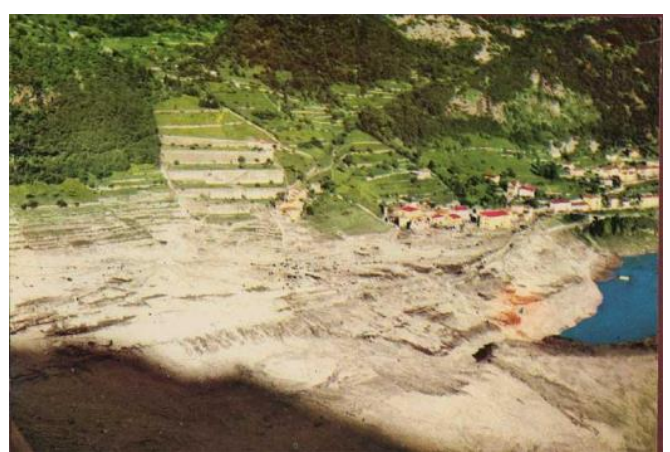

The town of Longarone after the dam was overtopped by a giant wave.

Figure 1-2. Shows the town of Longarone before and after the Vainot case occurred. (1963 Vajont Survivor's Committee Image source)

http://www.environmentandsociety.org/arcadia/expecting-disaster-1963-landslide-vajont$\underline{\mathrm{dam}}$ 


\subsection{Experimental study}

It is important to investigate and understand the impacts caused by tsunami waves. This can be accomplished by physical modeling in order to comprehend and refine the prediction methodology in mitigating tsunami hazards. Previous studies in numerical modeling of tsunami wave generation included rigid and deformable wedge sliding on a slope. Laboratory studies on landslide problems includes either two-dimensional (Fritz, 2002; Grilli and Watts, 2005; Heinrich, 1992; Wiegel, 1955), or three-dimensional experimentation (Enet and Grilli, 2007; Grilli and Watts, 2005; Liu et al., 2005). A wave can be generated by a rigid body sliding down a slope plane (Liu et al., 2005) or a landslide made by granular material (Watts, 1997). Wiegel (1955) introduced both the landslide masses falling vertically or sliding on an inclined slope under submerged conditions. The

author's results showed that the height of the generated waves relies on the initial depth of submerged block and the water depth. Additionally, the authors concluded that the wave period increases with the increasing length of the submerged block. However, the wave period decreases with a greater inclined slope angle. Liu et al. (2005) used a wedge with two different orientations and a hemisphere shaped wedge in their experiments. The initial condition of the landslide mass varied from fully aerial to totally submerged. Moreover, a range of sliding block with varying mass was used to alter the initial acceleration. Fritz (2002) conducted a series of experiments of deformable and rigid landslides with twodimensional laboratory model. His investigation was mainly focusing on the relevant factors of the impulsive waves including the weight of sliding masses, sliding speed, water depth and thickness of landslide masses. With a particular inclined angle $\left(45^{\circ}\right)$ through all his experiments, he mentioned that based on the Froude number and the dimensionless 
thickness of slide masses, four different types of wave can be observed including weakly non-linear oscillatory wave, non-linear transition wave, solitary-like wave, and dissipative transient bore. Ataie-Ashtiani and Nik-Khah (2008) conducted a series of experiments for both rigid and deformable material sliding on inclined slopes. Their investigations mainly focused on effective parameters that will affect the water surface and the impulsive wave characteristics. The major concern of their study was the inclined bed slope angle, geometry of the sliding blocks, velocity of the sliding blocks, and the water depth. Compared to previous experiments (Ataie-Ashtiani and Nik-Khah, 2008; Enet and Grilli, 2007; Watts, 1997; Wiegel, 1955), most of the research focused on the effective parameters of the altitude of impulsive wave; however, experimental research on the characteristics of the flow field is limited.

\subsection{Numerical study}

Mathematical analysis is also applicable to study engineering and science problems using the numerical approximation. In recent decades, the advance development of computing enhances its position and raise the use of numerical analysis in the engineering and science area. Engineers investigate the methods applied to the intractable problem, the strengths and pitfalls as well as the way to implement. Numerical analysis provides the efficiency, feasibility and flexibility to better collaborate with the research. In order to have a better result, researchers take adequate underlying models in the proper mathematical modeling of the processes.

A nonlinear Eulerian code, Nasa-Vof2D, has been used by Heinrich (1992) to study landslide tsunamis. This method solves incompressible Navier-Stokes equations by a 
mesh-based method. Liu et al. (2005) used the Smagorinsky subgrid model, which utilizes the Large Eddy Simulation (LES) method to study the three-dimensional sliding masses. Abadie et al. (2010) introduced a model using Direct Numerical Simulation (DNS) of Navier-Stokes equations to simulate two- and three-dimensional landslide tsunamis. The mesh-based method has shown good agreement on simulating the complex flows and the wave propagation (Grilli and Watts, 1999; Yuk et al., 2006) of tsunami waves. The meshbased methods, like finite difference method (FDM) and finite volume method (FVM) are based on the Eulerian approach that have evolved over decades to solve fluid problems. In addition, the Lagrangian method has been applied in both mesh-based and mesh-free methods. Compared to the mesh-based methods, the mesh-free methods connect points without a certain connectivity requirement (i.e. mesh). All physical properties have been carried and calculated within a particle instead of a mesh or a grid. Thus, the mesh-free methods are more robust in addressing the free surface problems. The two well-known mesh-free Lagrangian approaches are the Smoothed Particle Hydrodynamics (SPH) approach and the Moving Particle Semi-implicit (MPS) methods.

The SPH method was introduced by Lucy (1977) and Gingold,R.A. and Monaghan (1977) in astronomic studies. In the past few decades, the SPH method has been widely applied in many engineering fields. Monaghan and Kos (2000) applied the weakly compressible SPH (WC-SPH) method into a subaerial landslide study. Qiu et al. (2017) combined the WCSPH method and the Discrete Element Method (DEM) to simulate landslide tsunamis for both rigid and deformable sliding masses. Ataie-Ashtiani and Shobeyri (2008) introduced the implicit incompressible SPH (ISPH) method to study landslide generated waves for both rigid and deformable sliding masses. Yeylaghi et al. (2017) presented a parallel ISPH 
model to simulate the landslide generated wave for both subaerial and submarine landslide in two- and three-dimensions. The specific set-ups for the angle of the incline bed slope were $45^{\circ}$ for both rigid and deformable slides and vertical falling of a rigid box. The contour plot of the normal velocity showed the location of the vortex with a good agreement to the water surface as compared to the numerical and experimental results.

The MPS approach was first presented by Koshizuka and Oka (1996) to simulate fragmentation of incompressible fluids. Shakibaeinia and Jin (2009) developed the weakly compressible MPS (WC-MPS) approach for solving incompressible flow problems. Shakibaeinia and Jin (2012a, 2012b) employed multiphase model to simulate complex flows. Fu and Jin (2015) used the WC-MPS method to investigate landslide tsunamis for both rigid and deformable slides. A good corresponding result to the water surface changes can be found in their studies. However, their studies on landslide generated waves did not provide details of the flow pattern, in particular, of the velocity distribution in the simulation domain. Jin et al. (2016) used a similar method and illustrated detailed information of the flow fields to study the velocity distribution at certain locations for rigid landslide masses under three different water depth scenarios. However, the information was only available at the top edge of the sliding block. Details of the contour and vector plots were shown in the simulation domain. All of these investigations are on the landslide generated waves (Fu and Jin, 2015; Jin et al., 2016; Tajnesaie et al., 2018) on a specific bed slope angle of $45^{\circ}$.

\subsection{Scope of thesis}

The present study aims to present more detailed information on the landslide generated waves and the flow fields. The WC-MPS method is applied in this study to simulate 
landslide tsunamis at two different bed slope angles of $40^{\circ}$ and $50^{\circ}$. Sliding block released under water were studied by experimental data and compared to the numerical simulations. Chapter 2 will present the methodology of WC-MPS method. Chapter 3 will introduce the experimental set-up. The results comparing the numerical and experimental data will be illustrated in Chapter 4. Firstly, a validated comparison of the water surface will be presented and the simulated results of the velocity distribution along the sliding block is compared to the experimental measurements. The horizontal and vertical velocity distribution at the tail, the top surface and front toe of the sliding block are investigated in greater detail. Detailed flow information including the flow field information of both depthaveraged velocity distribution and contour plot is examined. The vorticity magnitude with the vector plot will represent the vortex movement. A summary of this work is discussed in Chapter 5.

\section{Chapter 2 : METHODOLGY}

\subsection{Governing equation}

The calculation of an incompressible Newtonian fluid flow is introduced by the continuity and momentum equations. Since the MPS is using the Lagrangian approach, the continuity equation with its vector form can be written as: 


$$
\frac{1}{\rho} \frac{D \boldsymbol{u}}{D t}=-\nabla \cdot \boldsymbol{u}
$$

and the momentum equation is described by

$$
\frac{D \boldsymbol{u}}{D t}=-\frac{1}{\rho} \nabla p+v \nabla^{2} \boldsymbol{u}+f
$$

where $\rho$ is the fluid density, $p$ is the pressure, $\boldsymbol{u}$ is the velocity vector, $f$ is the external force (i.e. gravity), $v$ is the kinematic viscosity, $\nabla$ is the gradient operator which will be introduced in section 2.4.

\subsection{Weighting function}

In the MPS method, the simulation domain is described by a set of disordered particles. An approach to distribute a particle's contribution that interacts with each other in its vicinity has been introduced as a kernel function (weighting function). In this paper, a third-order polynomial kernel function $W$ has been employed to the MPS method (Batchelor, 1967; Shakibaeinia and Jin, 2012b) as:

$$
W\left(r_{i j}, r_{e}\right)= \begin{cases}\left(1-\frac{r_{i j}}{r_{e}}\right)^{3} & r_{i j} \leq r_{e} \\ 0 & r_{i j} \geq r_{e}\end{cases}
$$

where $r_{i j}=\left|r_{i}-r_{j}\right|$ represents the distance between particle $i$ and $j, r_{e}$ describes the radius of the interaction area (Shakibaeinia and Jin, 2012b). In the MPS method, only the neighbouring particle $j$ will be calculated to interact with target particle $i$ in its interaction area. Therefore, the weighting function is to calculate the contribution of the neighbouring 
particles in a target particle's interaction circle. As Figure 2-1 showing, neighbouring particle $j$ will have a greater contribution when it is located closest to the target particle $i$. The objective of importing the kernel function is to smooth the physical quantities in the searching radius (Koshizuka et al., 1998; Koshizuka and Oka, 1996; Shakibaeinia and Jin, $2009,2012 b)$. Density of each particle has been defined as a dimensionless parameter. The particle number density has been defined as (Koshizuka et al., 1998; Shakibaeinia and Jin, 2009):

$$
\langle n\rangle_{i}=\sum_{j \neq i} W\left(r_{i j}, r_{e}\right)
$$

In an incompressible fluid, the density is constant. To satisfy the continuity equation, a constant particle number density has been introduced as $n^{0}$, which is the value at the initial condition.

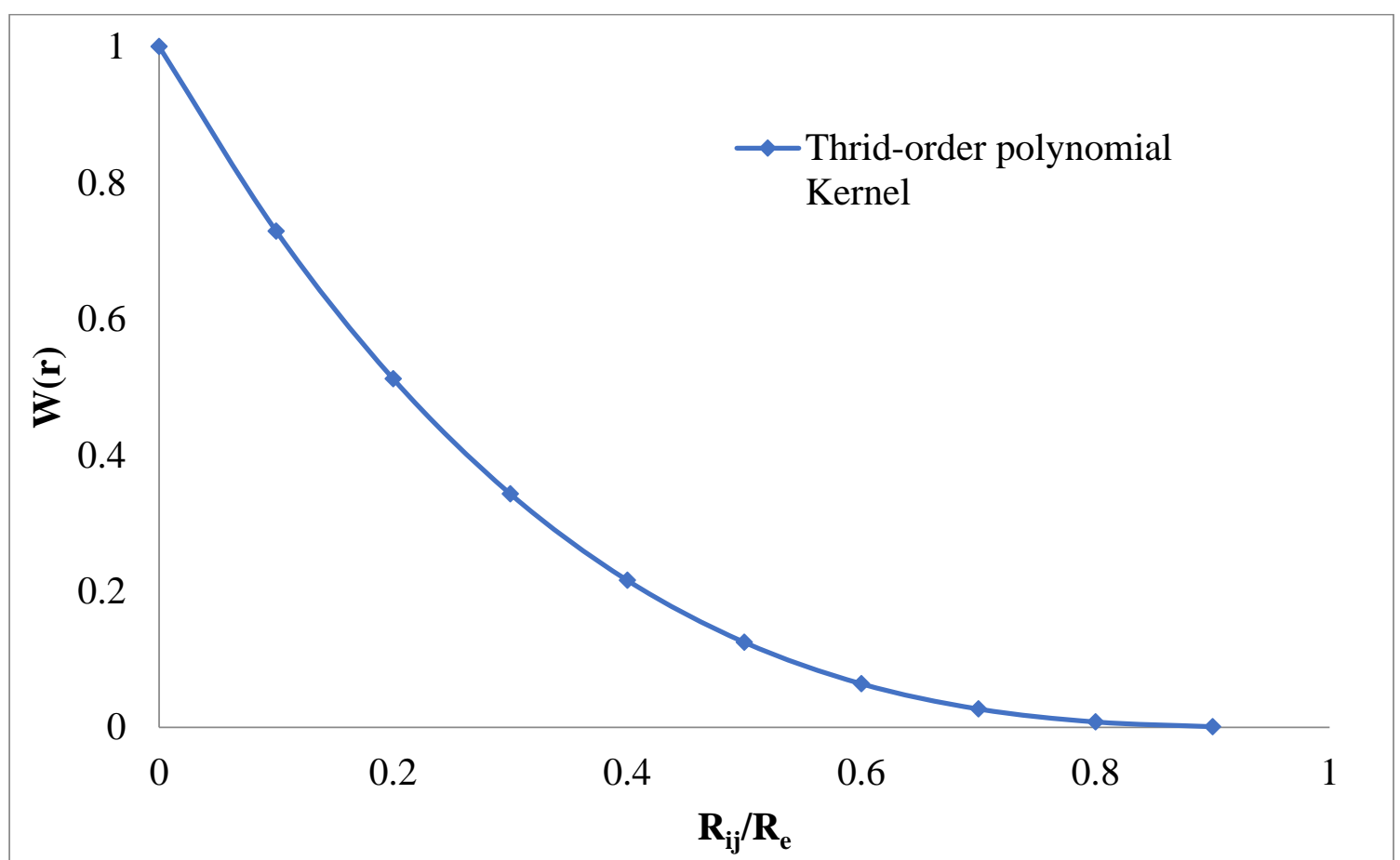

Figure 2-1. Kernel function 


\subsection{Neighbour search strategy}

As the previous section mentioned, in MPS approach, the physical properties of each particles are affected and calculated based on the values of neighbouring particles. Contrast to the mesh-based methods, which are considering the constant connecting between each cell among the simulated domain, the mesh-free (MPS or SPH) methods are looking for a changing of neighbouring of each particle at every time step. Therefore, an accurate and efficient strategy of searching neighbouring particles is needed and important for each particle. In the MPS methods, the particle $j$ will be considered as the neighbour particles of target particle $i$ when the distance between these two particles $\boldsymbol{r}_{i j}$ is less than assigned searching radius $\boldsymbol{r}_{\boldsymbol{e}}$. Koshizuka and Oka (1996) introduced the original algorithm in MPS method which is searching in the entire simulated domain to find the neighbouring for each particle; therefore, this algorithm has an operation of $N^{2}$, where $N$ is the total number of particles. Koshizuka et al. (1998) approached another algorithm to reduce the searching operations. Instead of using the neighbourhood radius as $r_{e}$, he increased the radius to $r_{e}+\delta$, where the $\delta$ is a small distance to confirm that all particles moved to the operational area of a certain particle and are considered as the neighbouring at next time step; also instead of building the neighbourhood at each time step, this algorithm updates the list of neighbours at every time step. Although, this strategy reduces the computational time, it still has a searching operation of $m N^{1.5}$. Gotoh et al. (2004) proposed another strategy known as "linked list algorithm". In this algorithm, the simulated domain is divided into square girds of a size $r_{e}$ based on a background Cartesian grid; while the searching area only aims on the located cell of the certain particle and its neighbouring eight cells as showing in Figure 2-2, the red-line area. In this study, the operated algorithm is collected 
form Shakibaeinia and Jin (2009), which combined the methods proposed by Koshizuka et al. (1998) and Gotoh et al. (2004). As showing in Figure 2-2, the grids are divided into a size of $r_{e}+\delta$ instead of a size of $r_{e}$. 


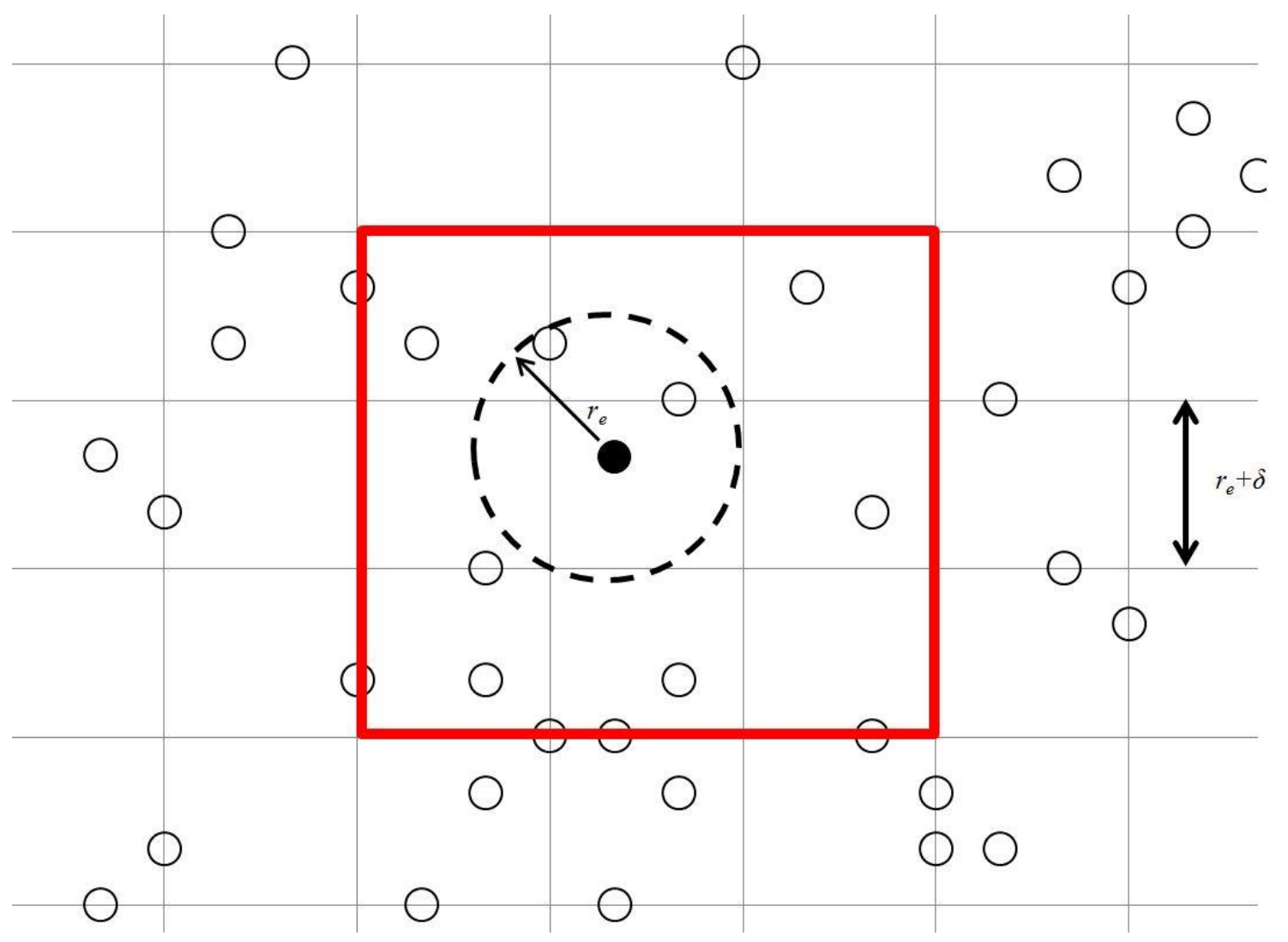

Figure 2-2. Sketch of searching neighboring particles algorithm.

\subsection{MPS discretization}

The first order gradient model was described by Koshizuka and Oka (1996), written as: 


$$
\langle\nabla \phi\rangle_{i}=\frac{d}{n^{0}} \sum_{j \neq i} \frac{\left(\phi_{j}-\phi_{i}\right)}{r_{i j}^{2}}\left(\boldsymbol{r}_{j}-\boldsymbol{r}_{i}\right) W\left(r_{i j}, r_{e}\right)
$$

where $d$ is the number of space dimensions; $\phi_{i}, \phi_{j}$ are the scalar quantities possessed by particles $i$ and $j$ respectively; $n^{0}$ is the initial particle number density. Thus, the gradient vector between particles $i$ and $j$ can be simply defined by $\left(\phi_{j}-\phi_{i}\right)\left(r_{j}-r_{i}\right) /\left|r_{j}-r_{i}\right|^{2}$ (Koshizuka and Oka, 1996). A gradient vector can be evaluated with any two particles. In MPS method, this model is specifically applied to calculate the pressure term. This approach is to satisfy the continuity equation and to avoid the clustering of particles (Koshizuka and Oka, 1996).

Similarly, the divergence model for vector $\boldsymbol{u}$ of the particle $i$ is described as:

$$
\langle\nabla \cdot \boldsymbol{u}\rangle_{i}=\frac{d}{n^{0}} \sum_{j \neq i} \frac{\left(\boldsymbol{u}_{j}-\boldsymbol{u}_{i}\right)}{r_{i j}^{2}}\left(\boldsymbol{r}_{j}-\boldsymbol{r}_{i}\right) W\left(r_{i j}, r_{e}\right)
$$

Another model given by the kernel function is the Laplacian model. Originally, it has been introduced from a transient diffusion problem (Batchelor, 1967; Colagrossi and Landrini, 2003; Koshizuka et al., 1998; Koshizuka and Oka, 1996; Xu and Jin, 2016).

$$
\left\langle\nabla^{2} \phi\right\rangle_{i}=\frac{2 d}{\lambda n^{0}} \sum_{j \neq i}\left(\phi_{j}-\phi_{i}\right) W\left(r_{i j}, r_{e}\right)
$$

where $\lambda$ is the correction coefficient used to minimize the concerns of the diffusion problems, and is introduced as (Batchelor, 1967; Koshizuka et al., 1998; Koshizuka and Oka, 1996; Shakibaeinia and Jin, 2009).

$$
\lambda=\frac{\int_{V} W\left(r_{i j}, r_{e}\right) r_{i j}^{2} d v}{\int_{V} W\left(r_{i j}, r_{e}\right) d v}
$$


The physical quantity lost by the particle $i$ is transferred to the particle $j$, which represents the conservation of the current Laplacian model (Koshizuka et al., 1998; Koshizuka and Oka, 1996; Shakibaeinia and Jin, 2009).

\subsection{Equation of State for pressure calculation}

In the MPS method, the equation of state is first introduced by Lee et al. (2011). In this paper, the equation of state is used to calculate the pressure in weakly compressible MPS (Shakibaeinia and Jin, 2009) instead of solving the Poisson equation. The equation of state has been modified by Shakibaeinia and Jin $(2009,2012 b)$ as:

$$
p_{i}^{n+1}=\frac{\rho c_{0}^{2}}{\gamma}\left(\left(\frac{\left\langle n^{*}\right\rangle_{i}}{n^{0}}\right)^{\gamma}-1\right)
$$

where $n^{*}$ is a temporal particle number density, thus it has been calculated based on the intermediate particle position $r^{*}$ (Koshizuka and Oka, 1996; Lee et al., 2011; Xu and Jin, 2016); $c_{0}$ is the sound speed, in order to satisfy a compressibility less than $1 \%$, it is given to ten times of the maximum speed in the simulation domain (Koshizuka and Oka, 1996; Shakibaeinia and Jin, 2012b; Tanaka and Masunaga, 2010); $\gamma=7$ is a widely used typical number in MPS method to simulate a weakly compressible flow (Fu and Jin, 2015; Koshizuka and Oka, 1996; Shakibaeinia and Jin, 2009; Xu and Jin, 2016).

\subsection{Boundary condition}

In order to avoid the fluid particles penetrating the solid boundary and the deficiency of the particle number density where particle near solid boundary, referred to as ghost particles, are introduced to the simulation domain (Koshizuka and Oka, 1996; Shakibaeinia and Jin, 2009). The velocity of the ghost particles is set to be 0 for the non-slip boundary. The pressure of the ghost particles is calculated for the first layer, which is equal to the pressure 
at the nearest boundary and transferred to other layers. The number of ghost particle layer is determined by the searching radius $r_{e}$. In this paper, a searching radius $r_{e}=3.5 \mathrm{DL}$ (DL is the average particle distance) has been used, and shown in Figure 2-3 as three layers of ghost particles. In Figure 2-3, the solid boundary and ghost particles are typically showing the initial condition of the inclined bed. Contrast to the regular setting of the bed which transfers the pressure of solid boundary directly vertical or horizontal to the ghost particles, the ghost particles behind the inclined solid boundary will obtain the pressure from the bed with the specific equation of slope angle which is showing as the dot-line in Figure 2-3. As Figure 2-4 showing, approaching to the free surface, the particle number density is deficient due to the non-existing fluid particles beyond the outer region. The determination of the free surface is defined by the density of particles as:

$$
\left\langle n^{*}\right\rangle_{i} \leq \beta n^{0}
$$

where $\beta$ is a coefficient with values ranging between 0.8 and 0.99 (Kondo and Koshizuka, 2011; Koshizuka et al., 1998; Koshizuka and Oka, 1996). In this paper, $\beta=0.97$ is used in the simulation model. Using Eq.(2.10), if the intermediate particle number density is less than $\beta$ times the initial particle number density, the particle is determined as a surface particle and the pressure of this particle is assigned to be zero (Koshizuka et al., 1998). 


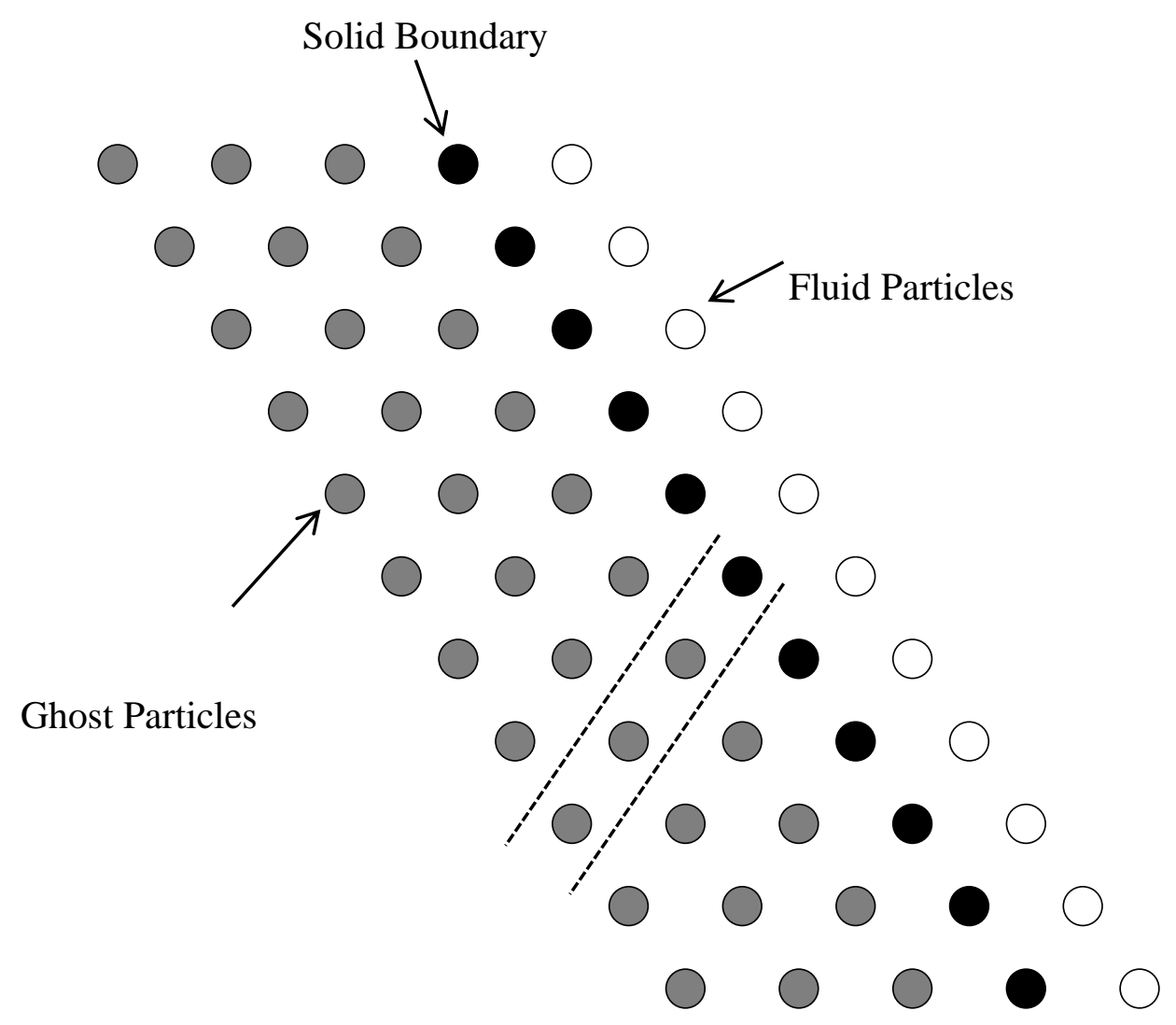

Figure 2-3. The solid boundary and Ghost particles 

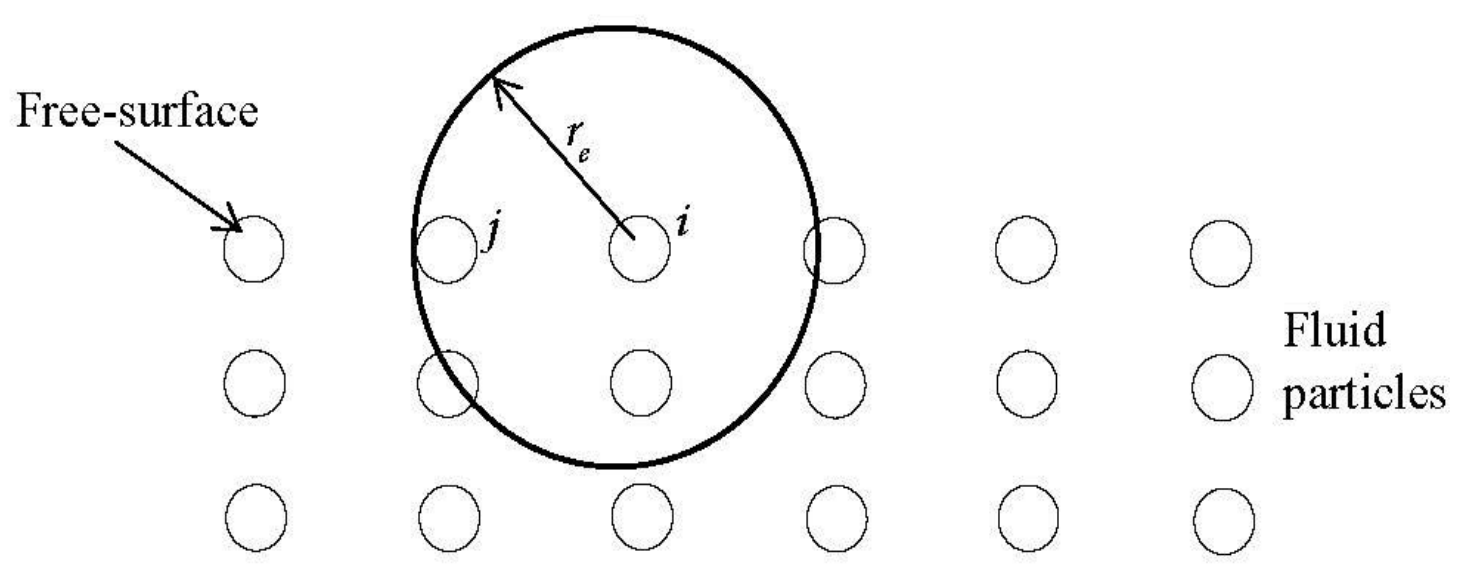

Figure 2-4. Free-surface

\subsection{Solution algorithm}

In the WC-MPS method, for solving the governing equation, the procedure is split in two phases related to the time interval. Typically, the two phases are named as prediction and correction (Shakibaeinia and Jin, 2009). In the prediction phase, the velocity is predicted by using the value of previous time step, and used for calculating the pressure. The pressure is added to the correction phase, and employed to correct the velocity for next time step. Accordance with the procedure, the physical properties of each particles of next time step (i.e. velocity and position) can be expressed as:

$$
\begin{aligned}
& u^{n+1}=u^{*}+u^{\prime} \\
& r^{n+1}=r^{*}+r^{\prime}
\end{aligned}
$$

Therefore, substitute Eq. (2.11) into the term Du/Dt, it can be rewrite as:

$$
\frac{\mathrm{D} \boldsymbol{u}}{\mathrm{D} t}=\frac{\boldsymbol{u}^{n+1}-\boldsymbol{u}^{n}}{\Delta t}=\frac{\boldsymbol{u}^{n+1}-\boldsymbol{u}^{*}}{\Delta t}+\frac{\boldsymbol{u}^{*}-\boldsymbol{u}^{n}}{\Delta t}=\frac{\boldsymbol{u}^{\prime}}{\Delta t}+\frac{\boldsymbol{u}^{*}-\boldsymbol{u}^{n}}{\Delta t}
$$


Where the $\left(^{*}\right)$ and $\left({ }^{\prime}\right)$ represent the values in prediction and correction phases respectively. $\boldsymbol{u}^{n+1}, \boldsymbol{u}^{n}, \boldsymbol{u}^{*}, \boldsymbol{u}^{\prime}$ are introduced by (Shakibaeinia and Jin, 2009) as the velocity in the next time step, velocity in the previous time step, predicted velocity, and corrected velocity, respectively. Therefore, substitute Eq. (2.13) into Eq. (2.2), the momentum equation can be rewritten as:

$$
\boldsymbol{u}^{\prime}+\left(\boldsymbol{u}^{*}-\boldsymbol{u}^{n}\right)=-\Delta t \frac{\nabla p^{n+1}}{\rho}+\Delta t \frac{\mu \nabla^{2} \boldsymbol{u}^{n}}{\rho}+\Delta t f
$$

Thus, it can be divided as:

$$
\boldsymbol{u}^{\prime}=-\Delta t \frac{\nabla p^{n+1}}{\rho}
$$

and

$$
\boldsymbol{u}^{*}=\boldsymbol{u}^{n}+\Delta t \frac{\mu \nabla^{2} \boldsymbol{u}^{n}}{\rho}+\Delta t f
$$

The Eq. (2.16) is used to calculating the velocity in prediction phase, while all the values are collected from the previous time step $(n)$. Recall the Eq. (2.12), the term $\boldsymbol{r}^{*}$ can be expressed as $\boldsymbol{r}^{*}=\Delta t \boldsymbol{u}^{*}$. After the intermediate particle position is defined, the temporal particle number density $\left(n^{*}\right)$ can be calculated as:

$$
\left\langle n^{*}\right\rangle_{i}=\sum_{j \neq i} W\left(\boldsymbol{r}^{*}, r_{e}\right)
$$

Thus, the pressure of the particles can be calculated using Eq. (2.9). To solve the pressure gradient term $\nabla p^{n+1}$ in Eq. (2.15), the gradient operator (Eq. (2.5)) is applied into the correction phase as: 


$$
\left\langle\nabla p^{n+1}\right\rangle_{i}=\frac{d}{n^{0}} \sum_{j \neq i} \frac{\left(p^{n+1}{ }_{j}-p^{n+1}{ }_{i}\right)}{r_{i j}^{2}}\left(\boldsymbol{r}_{j}-\boldsymbol{r}_{i}\right) W\left(r_{i j}, r_{e}\right)
$$

However, Koshizuka et al. (1998) discussed the instability of using this pressure gradient term into MPS method. An improvement of his works is to use the minimum pressure values among the neighboring particles of target particle $i$ instead of using the calculated pressure of the target particle $i$. Therefore, the Eq. (2.18) is expressed as:

$$
\left\langle\nabla p^{n+1}\right\rangle_{i}=\frac{d}{n^{0}} \sum_{j \neq i} \frac{\left(p_{j}^{n+1}-\hat{p}_{i}^{n+1}\right)}{r_{i j}^{2}}\left(\boldsymbol{r}_{j}-\boldsymbol{r}_{i}\right) W\left(r_{i j}, r_{e}\right)
$$

where the $\hat{p}^{n+1}{ }_{i}$ is equal to the minimum pressure $\left(\min p^{n+1}{ }_{j}\right.$ ) among its neighboring. The changing of positions of each particle at next time step (Eq. (2.12)) can be written as:

$$
\boldsymbol{r}^{n+1}=\boldsymbol{r}^{n}+\Delta t \boldsymbol{u}^{n+1}
$$

In this study, since the WC-MPS method uses an explicit time-splitting approach, the Courant-Friedrichs-Lewy (CFL) condition has to be satisfied in a stabilized condition. The CFL condition is introduced as:

$$
\frac{|\boldsymbol{u}|_{\max }}{D L} \Delta t<C
$$

where the $|\boldsymbol{u}|_{\text {max }}$ is the maximum velocity among the simulated domain, $D L$ is the particle distance, $C$ is the Courant number which is suggested between 0 to 1 . In this study, a Courant number $=0.4$ has been used to satisfy the steady condition.

The flowchart of entirely computational algorithm is showing in Figure 2-5. First of all, input the initial variables including the position, velocity, and pressure and calculated the initial particle number density $\left(n^{0}\right)$ based on the initial condition. Secondly, the list of 
neighbours and boundary conditions are set in each time step. Moving to the prediction stage, the predicted velocity is firstly calculated using external force (i.e. gravity), and diffusion terms, and the predicted position of each particle is calculated based on the predicted velocity. Then, the intermediate particle number density $\left(n^{*}\right)$ is computed, and the equation of state is employed to calculate the pressure. The gradient operator is deployed to calculate the pressure gradient, and the values are utilized to correct the velocity of each particle. At the end, the particle position, velocity, and pressure are assigned as the outputs in each time step, and as the input of next time step. 


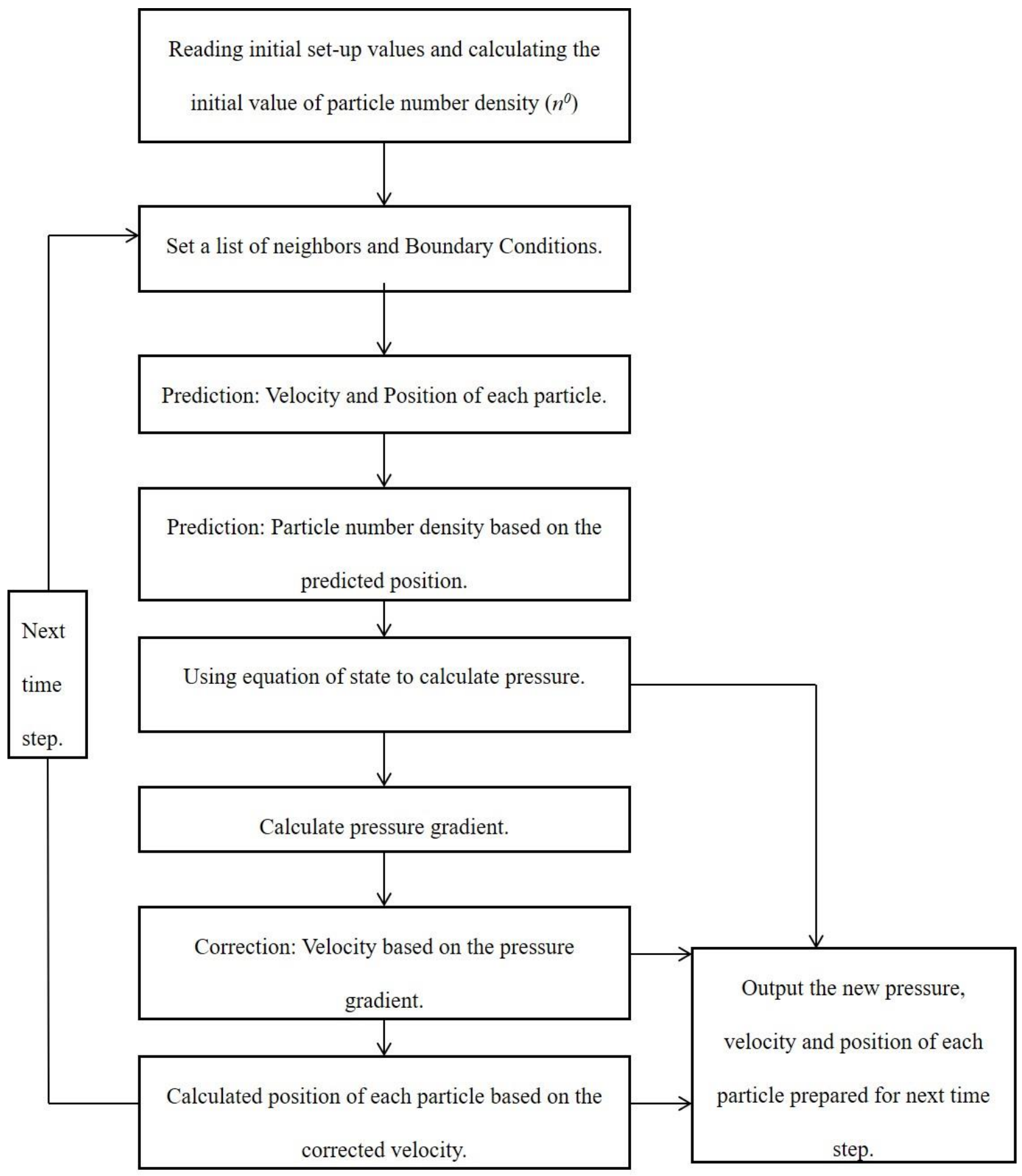

Figure 2-5. Flowchart of Computational algorithm. 


\section{Chapter 3 : EXPERIMENTS SET-UP}

In this study, the experiments are conducted at the National Cheng-Kong University in Taiwan. A high-speed camera (with 500 frames per second) is used for capturing the pictures in experiments, and the Digital Particle Image Velocimetry (DPIV) (Willert and Gharib, 1991) technique is employed for estimating the instantaneous velocity distribution of flow field the velocity fields. The open source package, PIVlab (Thielicke and Stamhuis, 2014), served as the major technical tool by analyzing the snapshots for extracting the velocity fields. Figure 3-1 is a series of snapshots showing an overall implementation of the experiment, where the bottom of the picture is parallel to the inclined slope. In Figure 3-1, to represents the initial time when the experiment started.

(a)

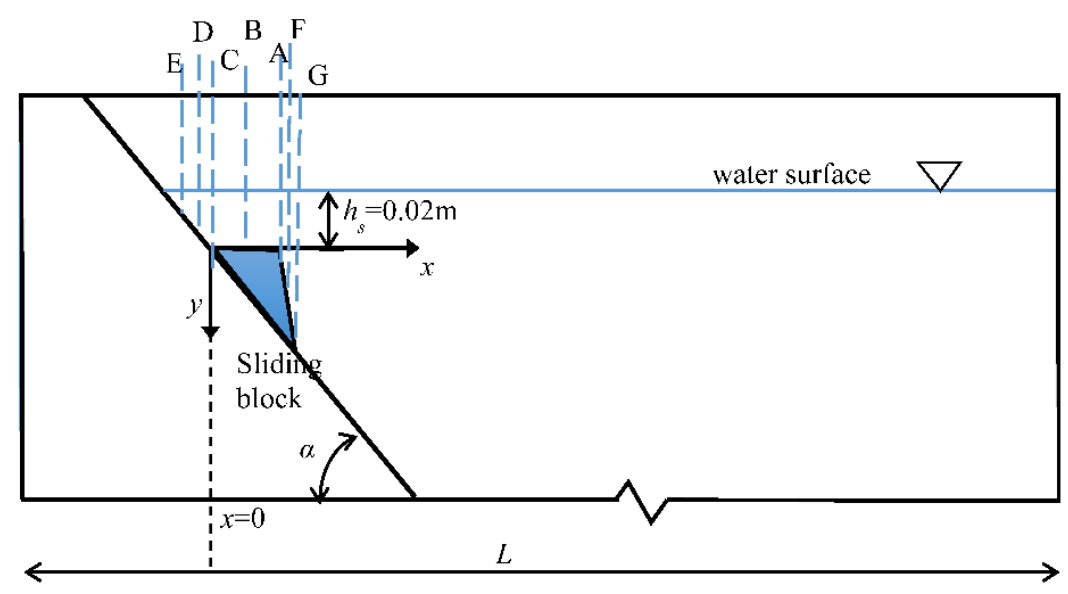

(b)

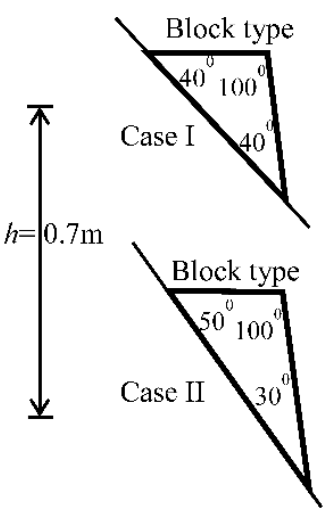

Figure 3-2Figure 3-2(a) shows the initial set-up of the experiments, where the sliders have been set as $0.02 \mathrm{~m}$ below the water surface, and the tank is filled with $0.70 \mathrm{~m}$ of water. As shown in panel (b), there are two types of sliding block. The bed slope angle $\alpha$ is related to the angle of block, that the top edge is set to be parallel to the water surface. Velocity distributions above the slider are investigated, where vertical sections A, B, C, D, E, F and 
G are assigned for case I, and A, C, D, E, and G for case II. The sections A, C, G are at the three corners of the slider. $\mathrm{B}$ and $\mathrm{F}$ are at the middle of the top edge and in front boundary respectively. D, E are selected on the bed, and separated $0.02 \mathrm{~m}$ away. In order to measure constantly, the original point of the coordinate system has been set at point $\mathrm{C}$. The sliding block is made of metal filled with steel beads. The slider of the case I has a volume $248.82 \mathrm{~cm}^{3}$ with a weight of $728.5 \mathrm{~g}$, and the case II uses a slider of $268.482 \mathrm{~cm}^{3}$ with a weight of $766.0 \mathrm{~g}$. The specific gravity is calculated as 2.928 (case I) and 2.853 (case II). Due to the image resolution and limited memory size of the camera, the experimental data are only available for the first $0.4 \mathrm{~s}$. The other experimental data are summarized and listed in Table 3-1, in which $S$ denotes the moving distance of the block along the inclined bed. It should be noted that the sliding speed is not under a steady acceleration. In the numerical simulations, the location of the slider is well controlled to make sure that the slider's position will be exactly the same as experiments.

\begin{tabular}{|c|c|c|c|c|c|c|}
\hline & Slope $\alpha$ & $h_{s}(\mathbf{m})$ & $h(\mathbf{m})$ & Time (s) & $S(\mathrm{~m})$ & $U_{b l k}(\mathrm{~m} / \mathbf{s})$ \\
\hline \multirow{5}{*}{$\begin{array}{c}\text { CASE I } \\
\mathbf{4 0}^{\circ} / \mathbf{4 0} \% / 100^{\circ}\end{array}$} & \multirow{5}{*}{$40^{\circ}$} & \multirow{5}{*}{0.02} & \multirow{5}{*}{0.70} & 0 & 0 & 0 \\
\hline & & & & 0.1 & 0.018 & 0.181 \\
\hline & & & & 0.2 & 0.058 & 0.399 \\
\hline & & & & 0.3 & 0.121 & 0.630 \\
\hline & & & & 0.4 & 0.190 & 0.693 \\
\hline \multirow{5}{*}{$\begin{array}{c}\text { CASE II } \\
\mathbf{3 0}^{\circ} / \mathbf{5 0} \% / 100^{\circ}\end{array}$} & \multirow{5}{*}{$50^{\circ}$} & \multirow{5}{*}{0.02} & \multirow{5}{*}{0.70} & 0 & 0 & 0 \\
\hline & & & & 0.1 & 0.024 & 0.236 \\
\hline & & & & 0.2 & 0.073 & 0.499 \\
\hline & & & & 0.3 & 0.140 & 0.663 \\
\hline & & & & 0.4 & 0.216 & 0.763 \\
\hline
\end{tabular}

Table 3-1. The experimental setup of case I and case II 

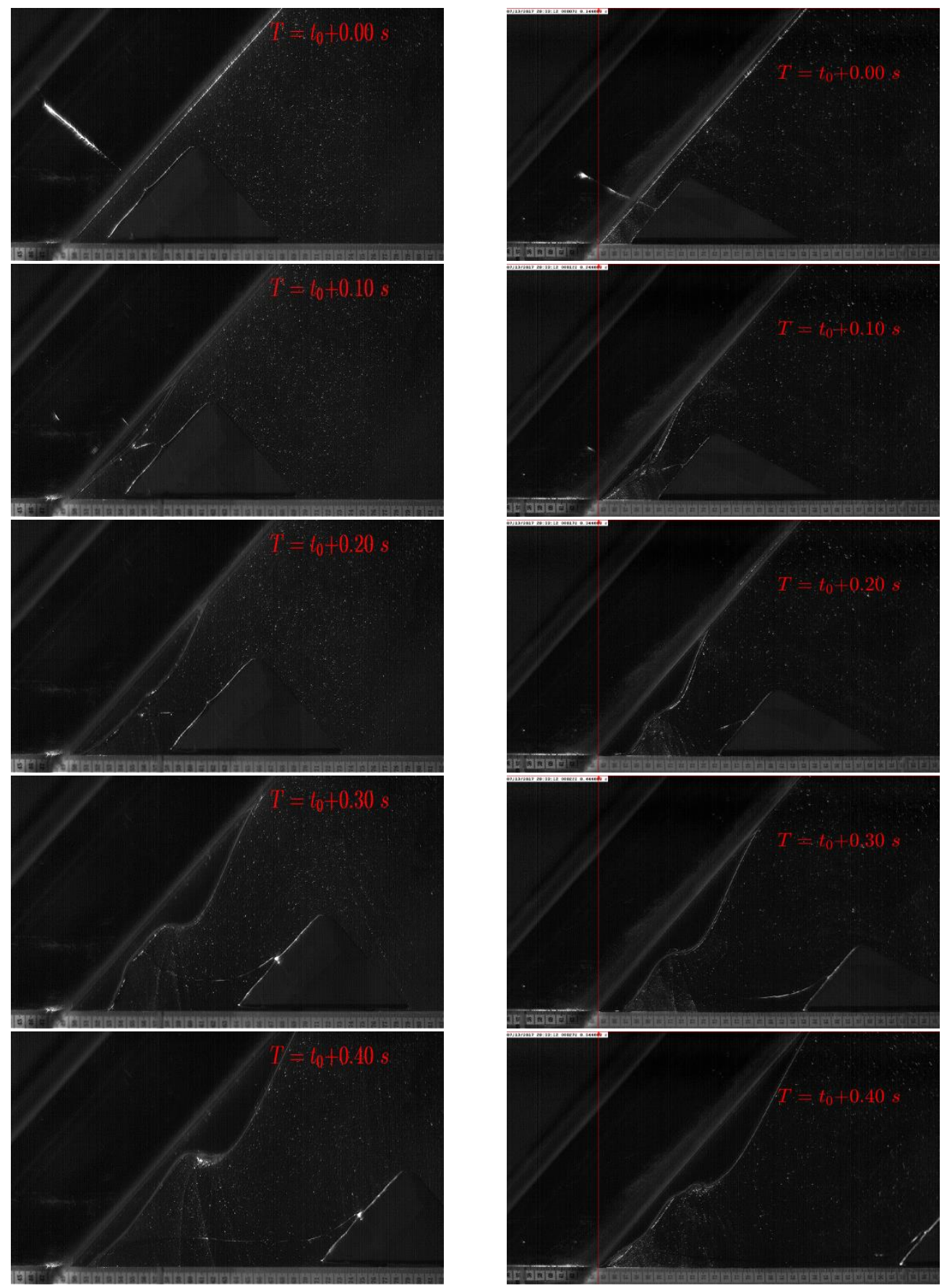

a

b

Figure 3-1. Snapshots during the experiments. (a) case I, where the inclined slope angle is $40^{\circ}$; and (b) case II with bed slope angle $50^{\circ}$ 

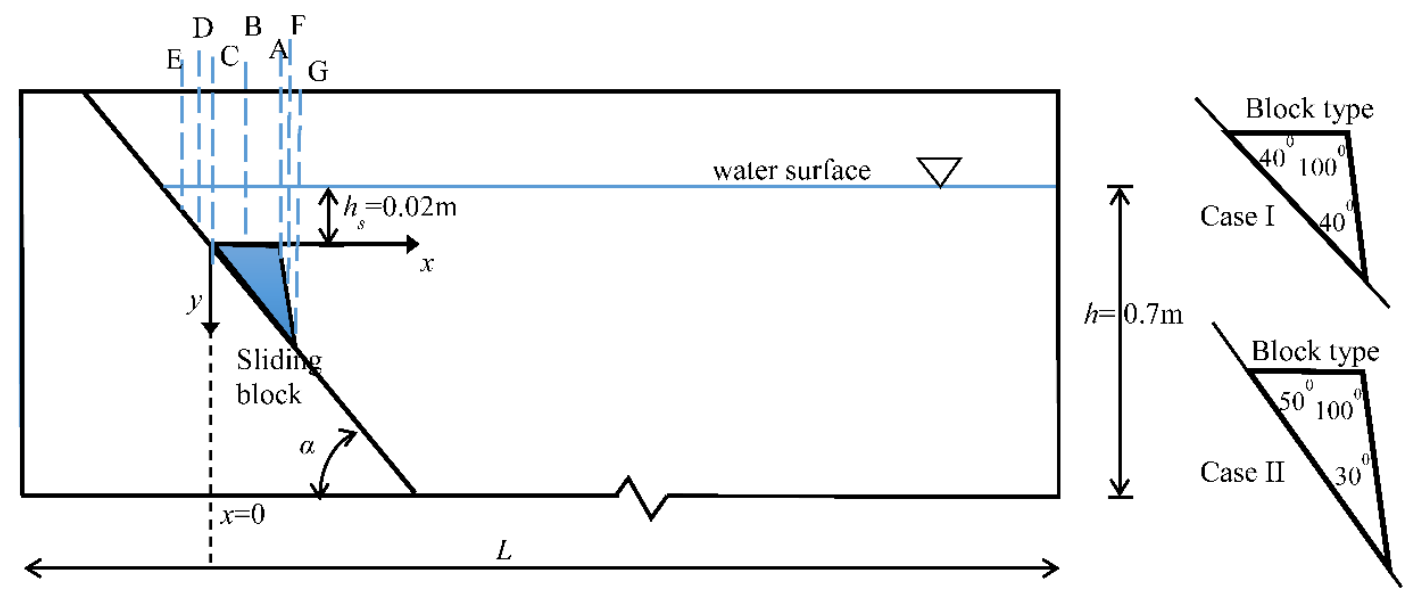

Figure 3-2. (a)The initial condition of experimental set-up. (b) Two different block types. 


\section{Chapter 4 : RESULT AND DISCUSSION}

The WC-MPS model is used to simulate submerged rigid landslides. The water has an assigned density of $1,000 \mathrm{~kg} / \mathrm{m}^{3}$, and the sliding blocks are treated as a solid with densities of $2,928 \mathrm{~kg} / \mathrm{m}^{3}$ and $2,853 \mathrm{~kg} / \mathrm{m}^{3}$ for case I ( $40^{\circ}$ inclination angle) and case II $\left(50^{\circ}\right.$ inclination angle) respectively. A time interval of $2.5 \times 10^{-5} \mathrm{~s}$ was used in the simulations. The length of the tank for case I is $2.19 \mathrm{~m}$ length, and $1.84 \mathrm{~m}$ for case II. The difference in the length of the tank will have minimal effects on the wave generation as the focus of this study is the detailed examination of the flow fields surrounding the sliding block at the two inclination angles. With particle distance of $0.005 \mathrm{~m}$ for both case I and II, the total particle number would be 42,336 and 39,416 for case I and II, respectively. The computation time for both cases is $0.4 \mathrm{~s}$.

\subsection{A validation of water-surface}

The WC-MPS method is used to simulate the free surface of the water representing deformation from landslides. In order to compare the results, the $x$-axis is along the bed direction, and the $y$-axis is vertical to the bed. In Figure 4- 1 and Figure 4-2, the dotted-line represents the reference location at the top edge of the sliding block illustrating the movement and location of the impulsive wave at each time step.

\section{Case I:}

The case I scenario represents a metal block sliding on a $40^{\circ}$ inclination slope. Figure 4-lillustrates the comparison between the experimental and the simulated results using WC-MPS. In the immediate stage of block release at $t=0.1 \mathrm{~s}$ and $0.2 \mathrm{~s}$, the water surface elevation showed good agreement between the experimental data and the simulated results. However, at $t=0.3 \mathrm{~s}$, the data showed an impulsive wave and the simulated results was able 
to generate the wave behavior. The sudden change in the water level was represented by the WC-MPS method. At $t=0.4 \mathrm{~s}$, deviations in the wave depth can be observed with a $10 \%$ difference between the experimental to the numerical results. The water surface levels showed good agreement at the tail end of the wave generation at all time steps, likely due to the fact that wave generated by the sliding block has yet to have an effect in the generation of deformation in the free surface particles within the limited timeframe of $0.4 \mathrm{~s}$.

\section{Case II:}

An inclination angle of $50^{\circ}$ represents the case II scenario. Figure 4-2 illustrates the comparison between the experimental to the simulated results. Similar to the case I findings, good agreement between the experimental and simulated results of the water level is observed at $t=0.1$ and $0.2 \mathrm{~s}$. An impulse wave also occurred at $t=0.3 \mathrm{~s}$ at the same location as in the case I scenario. However, the water level at the back of the sliding block showed an estimated error of $5 \%$ with the simulated results showing a lower surface water level as compared to the experimental dataset. The lower simulated water level could be caused by an inaccurate slider motion equation or pressure fluctuation. Similarly, good agreement between the experimental and simulated results were observed at $t=0.4 \mathrm{~s}$. As shown in case I, differences at the location of the wave bottom can be detected. In general, the results showed good agreement between the experimental and the WC-MPS simulated results in its ability to represent the deformation behavior of landslides. 


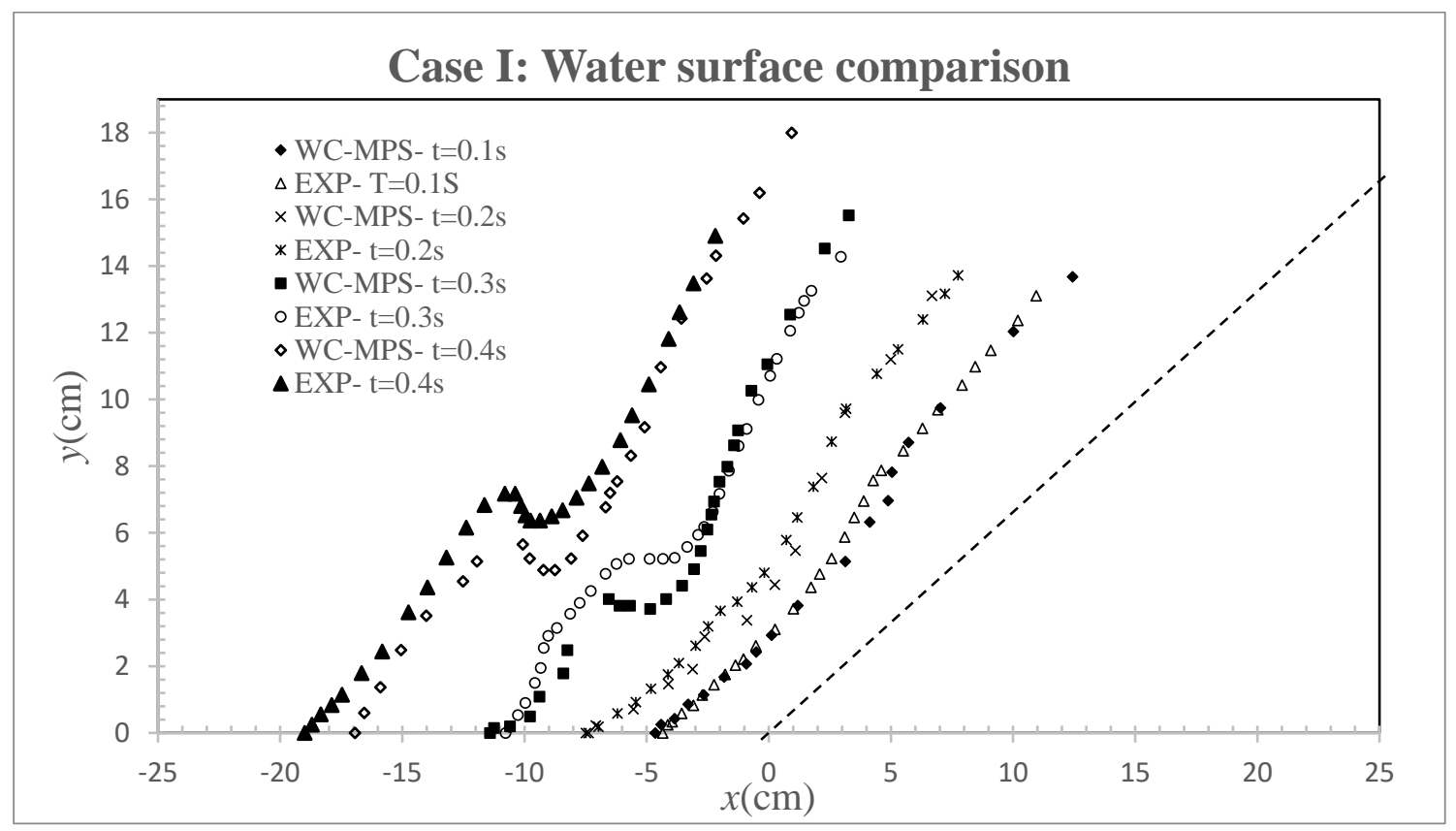

Figure 4-1. Case I: the water surface comparison between simulation result and experimental data. 


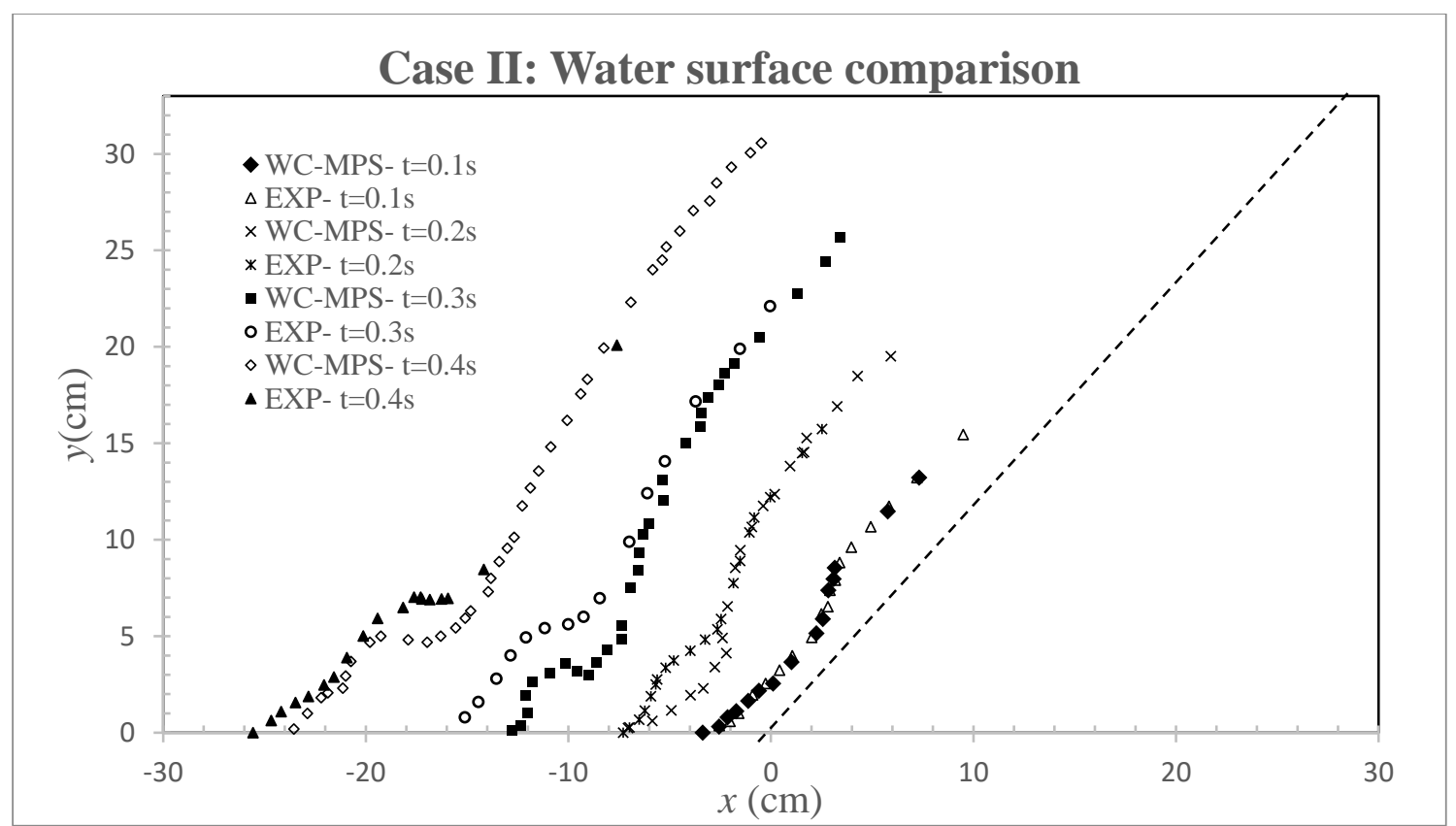

Figure 4-2. Case II: the water surface comparison between simulation result and experimental data.

\subsection{Velocity distribution}

The velocity distribution profile was analyzed for case I and case II of the sliding block. Experimental results were compared to the simulated WC-MPS method to represent the free surface flow. In the interpretation of the dataset, the horizontal axis is normalized by dividing the horizontal $(u)$ and the vertical velocity $(v)$ to the block velocity $U_{b l k}$ (Table 
3-1Error! Reference source not found.). Similarly, the vertical axis is normalized by dividing the depth of the selected points $(h)$ to water depth at the specified location $(H)$. For the velocity component in the vertical direction, positive value is interpreted as the wave is moving in an upward direction. For the velocity component in the horizontal direction, a positive value means the wave is moving to the right-hand side whereas a negative value means that it is moving towards the left-hand side or the shore direction.

\section{Case I:}

For the case I scenario, details of the velocity distribution at cross sections A, B, C, D, E, and $\mathrm{F}$ were analyzed and simulated using WC-MPS (Figure 3-2 (a)). In the initial time step as the block slides down the $40^{\circ}$ inclination plane, there is a negative effect on the water level surface as evidenced by the negative development of the vertical velocity near the water surface at cross section A. Figure 4-3 illustrates the velocity distribution profile of the sliding block at the $40^{\circ}$ inclination slope showing a positive displacement and transitioning to become negative near the water surface at $t=0.1 \mathrm{~s}$. The experimental result of the horizontal velocity shows a negative distribution; however, the numerical result shows a positive distribution near the edge of the block and alters to a negative distribution near the water surface. At $t=0.2 \mathrm{~s}$, both the experimental and numerical results show a similar trend. Similarly, the velocity profile and magnitude showed good agreement at $t=0.3$ and $0.4 \mathrm{~s}$ in both the vertical and horizontal directions. In general, the vertical and horizontal distribution profile at cross section A showed good agreement.

Figure 4-4 shows the velocity distribution at cross-sections F, located at the midpoint of the front surface and G, at the bottom of the sliding block. Due to the limitation of the camera view angle, the data was not available for cross-section $\mathrm{F}$ for $t>0.3 \mathrm{~s}$, and $\mathrm{G}$ at $t=0.3 \mathrm{~s}$. 
At cross-section F and G, horizontal and vertical velocities of experimental and numerical showed good agreement. At cross-section F, the simulation result showed some fluctuations on the horizontal velocity profile at $t=0.1$. This may be caused by the unstable particle movements at the beginning of simulation. The experimental data displays some fluctuations near boundary of the block at $t=0.3 \mathrm{~s}$ for the horizontal and vertical profiles.

For cross section G, similar velocity profiles are observed on the vertical flow. After the simulation domain becomes stabilized, the simulation results show good comparison to the experimental data on both horizontal and vertical direction. Stabilization on the horizontal velocity profile can be seen in Figure 4-4 (e). The horizontal velocity profile shows a positive direction development under $Y=0.5$ and gradually becomes negative. An opposite distribution can be identified on the vertical velocity profile, where it starts as a negative development and generally transitions to become a positive displacement.

Cross-section E is located immediately below the water surface and its horizontal and vertical velocity profiles illustrate that the value is close to zero at $t=0.3 \mathrm{~s}$ (Figure $4-5 \mathrm{a}, \mathrm{b}$ ). The results indicate that there are little effects on the velocity profile at this point on the sliding block. The data shows more detailed information at $t=0.4 \mathrm{~s}$.

Figure 4-5 (c) and (d) illustrates the velocity profiles at cross section D. The $u$ velocity profile at $t=0.3 \mathrm{~s}$ is similar to the profile at section $\mathrm{E}$ at $t=0.4 \mathrm{~s}$, while the vertical velocity profile has been affected by the movements of the sliding block by showing a negative (downward) flow direction. The vertical velocity changes to the positive direction near the water-surface. The change in velocity can be explained as the front of the impulsive wave starts moving backward to push the water surface level upwards with the second vertex appearing at $t=0.4 \mathrm{~s}$ at cross-section D. Compared to these two results, a similar distribution 
can be observed for both horizontal and vertical velocity profile. The simulation result illustrates good agreement for the vertical velocity profile at $t=0.4 \mathrm{~s}$. The comparison of the horizontal velocity profile, however, shows an opposite direction near the bed.

Figure 4-6 (a, b) illustrates the horizontal and vertical velocity profiles at cross section C, located at the left corner of the sliding block. The profiles show that the horizontal velocity transitions from positive to negative direction at $t=0.3 \mathrm{~s}$ and $t=0.4 \mathrm{~s}$. The comparison between experimental and WC-MPS results show a difference for the horizontal flow velocity component between $Y=0$ to $Y=0.5$ at $t=0.4 \mathrm{~s}$. At this corner, the flows are affected by the slope angle and the sliding block. Meanwhile, the water flow in this area is likely disturbed by differences in the pressure. The WC-MPS method, however, still shows good agreement on its simulation results for solving the free water surface, and the velocity component and distribution at $t=0.4 \mathrm{~s}$. At cross-section $\mathrm{B}$, good agreement between the experimental and simulated results in both horizontal and vertical velocity profiles as illustrated in Figure 4-6 (c, d). Similar velocity distributions in both experimental and numerical results is an indication of the robust capability of the WC-MPS method in simulated flows in complex areas. 

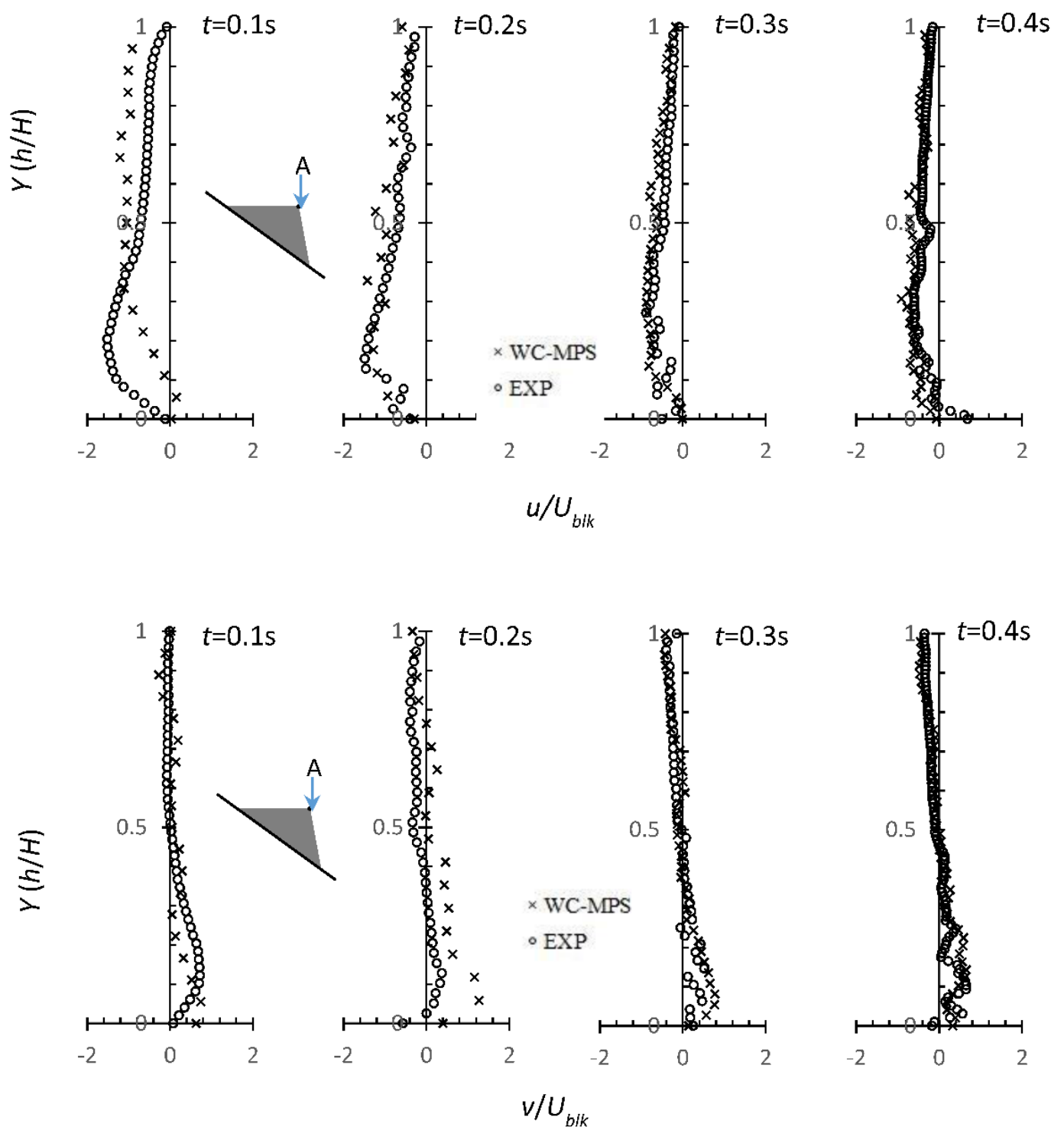

a

b

c

d

Figure 4-3. Comparison of velocity distribution at the top corner of the slider (crosssection $\mathrm{A})$ at $\mathrm{t}=0.1 \mathrm{~s}, 0.2 \mathrm{~s}, 0.3 \mathrm{~s}, 0.4 \mathrm{~s}$ respectively. 


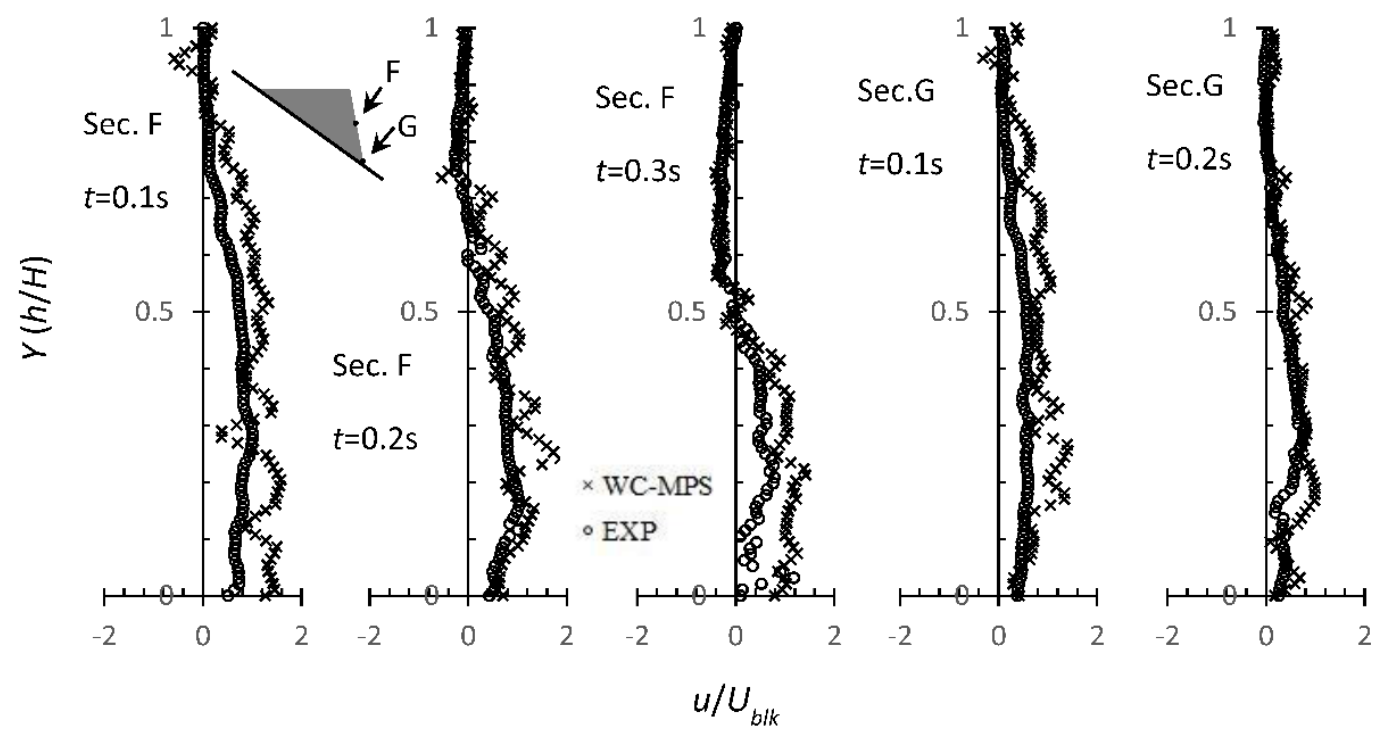

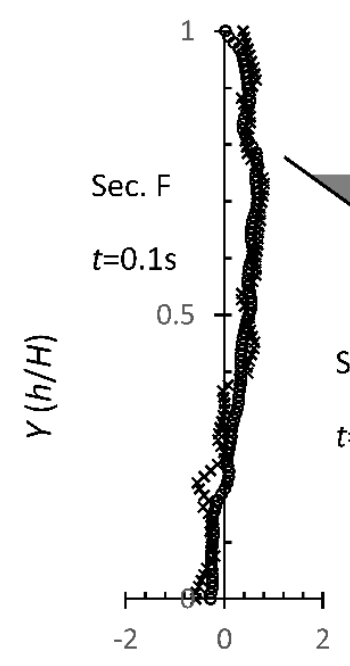

a

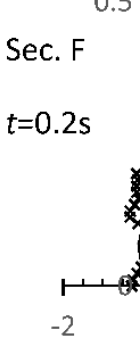

b

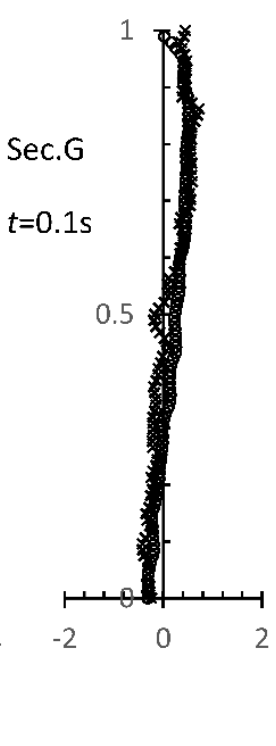

d

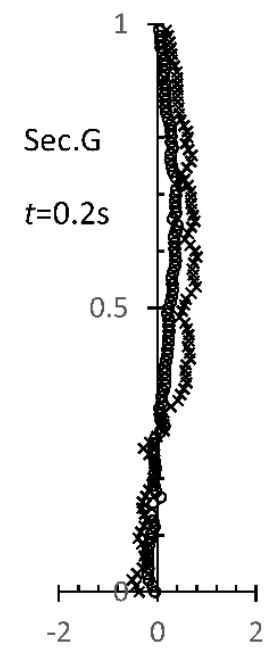

e

Figure 4-4. The comparison of velocity distribution at the cross-section $G$ and F. The comparison is available for cross-section $\mathrm{F}$ at $t=0.1 \mathrm{~s}, 0.2 \mathrm{~s}, 0.3 \mathrm{~s}$ and $\mathrm{G}$ at $t=0.1 \mathrm{~s}$ and $0.2 \mathrm{~s}$. 

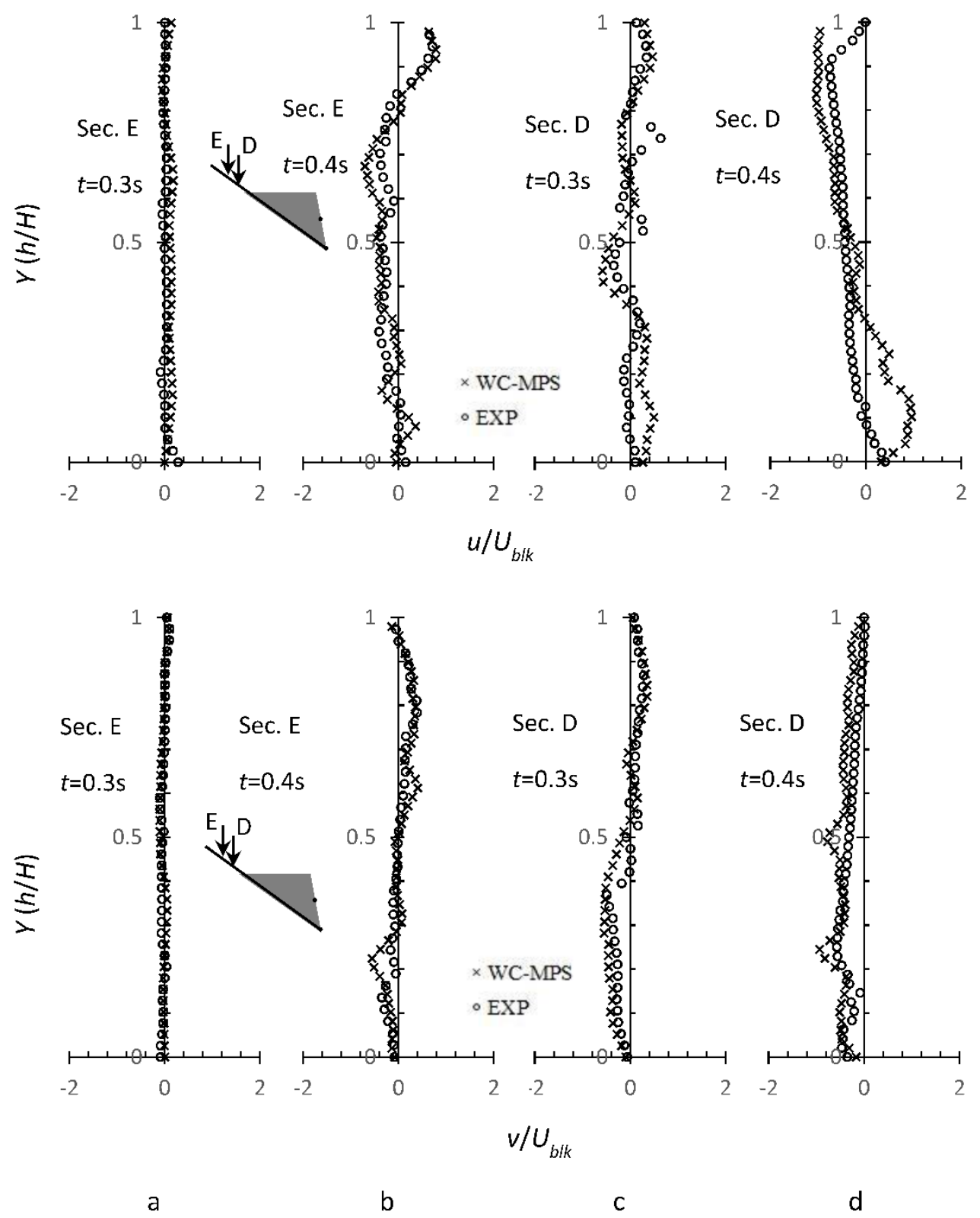

Figure 4-5. The comparison of velocity distribution for the cross-section D and E at $t=0.3 \mathrm{~s}$ and $0.4 \mathrm{~s}$. 

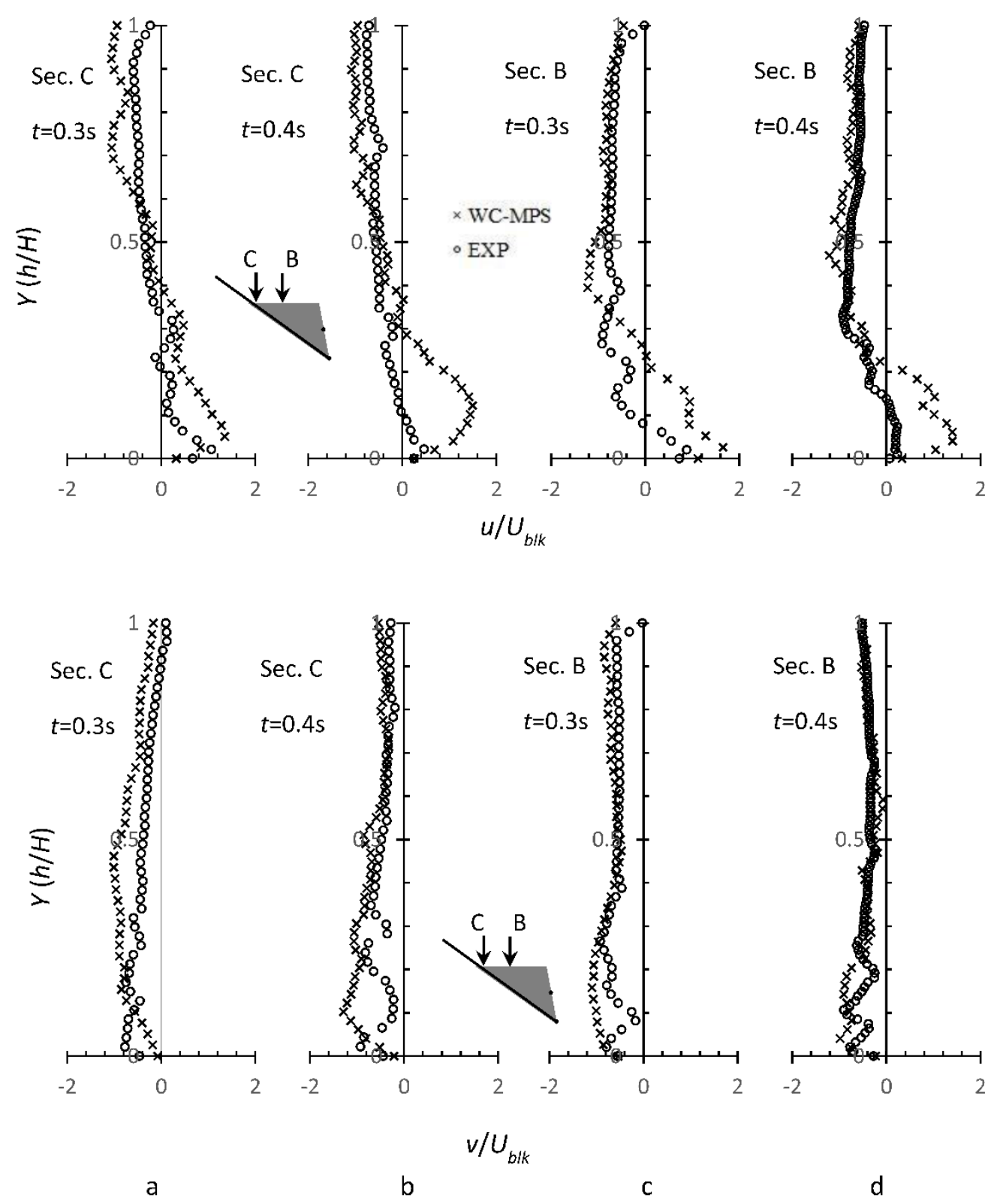

Figure 4-6. The comparison of velocity distribution for the cross-section C and B at $t=0.3 \mathrm{~s}$ and $0.4 \mathrm{~s}$. 


\section{Case II:}

Case II represents a sliding block on an inclination slope of $50^{\circ}$. Cross sections A, C, D, E, and $\mathrm{G}$ were analyzed in greater detail to evaluate the characteristics of the free surface from the sliding block. Figure 4-7 (a, b, c) illustrates the velocity distribution at cross sections A located at the top corner of the sliding block at $t=0.1,0.2$, and $0.3 \mathrm{~s}$. At $t=0.1 \mathrm{~s}$, instability is highlighted in the simulation domain. The simulation result of the horizontal velocity component shows a difference between $Y=0$ to $Y=0.5$. A similar distribution can be observed for the vertical velocity distribution; however, the numerical and experimental result are represented as two distinct lines. At $t=0.2 \mathrm{~s}$, the simulation result becomes more stable, hence, both horizontal and vertical velocity distribution show a good agreement on its trend and value. At $t=0.3 \mathrm{~s}$, a constant distribution of the water flow occurred for both the horizontal and vertical velocity components. As compared to the velocity distribution from case I, the distribution profiles from case II results shows a similar trend and value. In general, the development of a similar distribution in both cases suggests the velocity distribution is independent of the slope angle within the limited timeframe.

Figure 4-7 (d, e, f) illustrates the velocity distribution at cross-section $G$ located in the lower corner of the sliding block. The results show minor discrepancies between the simulated and experimental results in both the horizontal and vertical distribution profiles at $t=0.1 \mathrm{~s}$. The velocity distribution becomes stabilized at $t=0.2 \mathrm{~s}$, transitioning to become relatively constant distribution at $t=0.3 \mathrm{~s}$. Experimental data illustrates minor fluctuations at $t=0.2$ and $t=0.3 \mathrm{~s}$. Compared to case $\mathrm{I}$, cross-section $\mathrm{G}$ shows a similar velocity distribution at $t=0.3$ when it becomes stable. A similar comment can also be made for this point that the bed slope angle will have less effect at the front area of the sliding block. 
Figure 4-8 $(\mathrm{a}, \mathrm{b})$ illustrates cross section $\mathrm{E}$, where development of the flow pattern is occurring on the shore. The horizontal velocity component shows good agreement between simulated and experimental results. Development of the trend in the velocity distribution transitions from a positive to a negative direction. Due to the $50^{\circ}$ bed slope, the development of bubbles or air entrapment in the water may occur near the sliding block as demonstrated by slight fluctuations of the free surface. The simulation shows good stabilization condition near the edge of the sliding block for both horizontal and vertical velocity profiles.

In Figure 4-8 (c, d), a similar fluctuation to cross section $\mathrm{E}$ on the experimental measurement is also illustrated at cross-section D. For the vertical direction, the experimental data shows significant instability between $Y=0$ to $Y=0.5$ at $t=0.3 \mathrm{~s}$. In addition to good agreement on the development of velocity trend, the values between the simulated and experimental results are similar. At cross-section D, the simulation and experimental result illustrates a similar trend at $t=0.4$.

Figure 4-8Figure 4-8 (e, f) illustrates cross-section $\mathrm{C}$ located at the left corner of the sliding block. The velocity distribution profile shows close agreement between the simulated and experimental results. Discrepancies occurring between $Y=0$ to $Y=0.5$ may be due to the effect of pressure gradients generated during the instantaneous movement down the inclined plane resulting in an unsteady distribution near the boundary of the sliding block along the horizontal plane. However, good agreement was observed in both the horizontal and vertical profile near the water surface level at $t=0.4 \mathrm{~s}$. As compared to case I, both experimental and numerical results show similar vertical and horizontal distribution 
profiles. In general, the velocity distribution indicate that the horizontal and vertical profiles are similar at any inclination bed slope angles within the limited timeframe. 

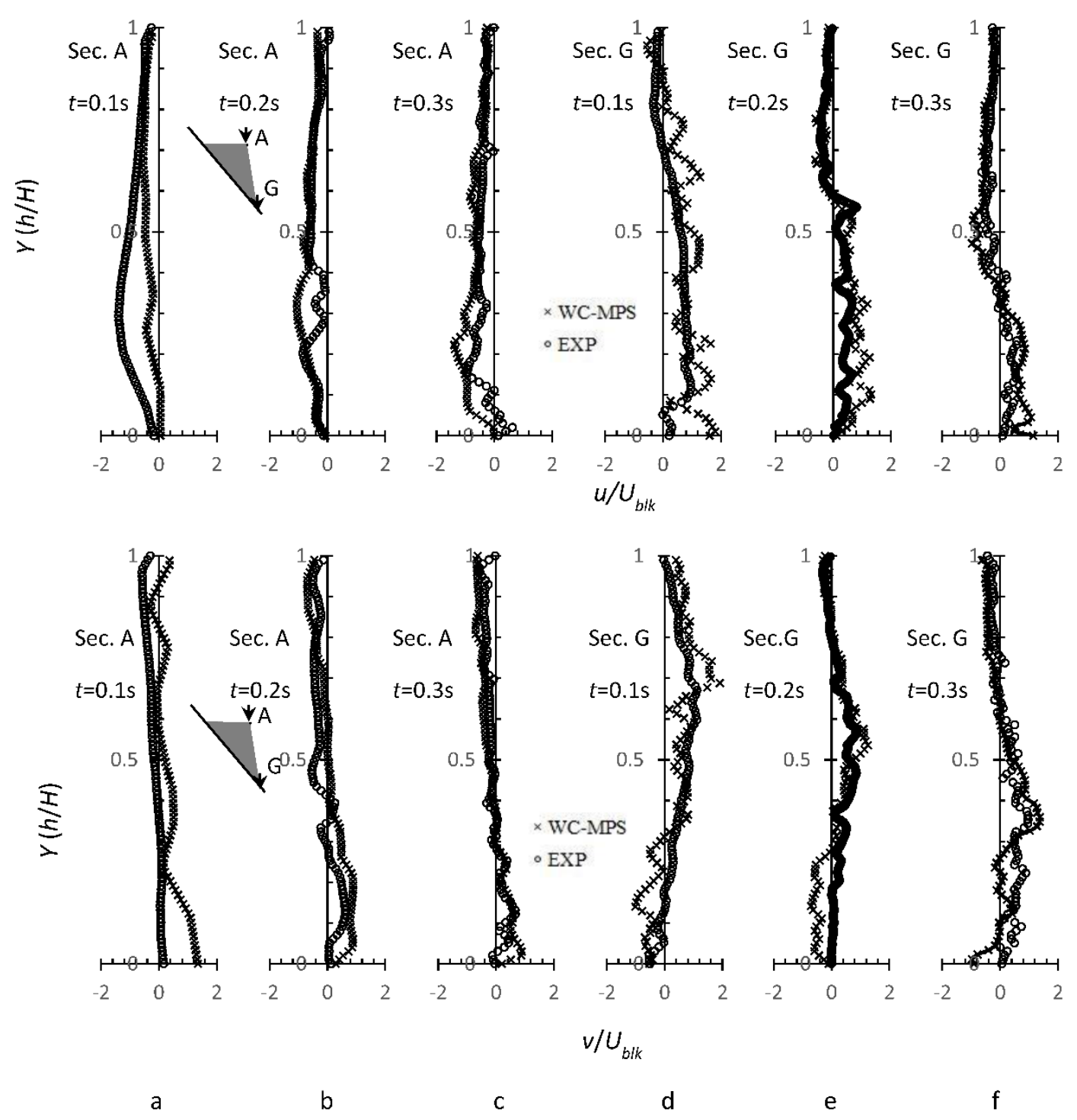

Figure 4-7. The comparison of velocity distribution for the cross-section A and G of Case II at $t=0.1 \mathrm{~s}, 0.2 \mathrm{~s}$, and $0.3 \mathrm{~s}$. 

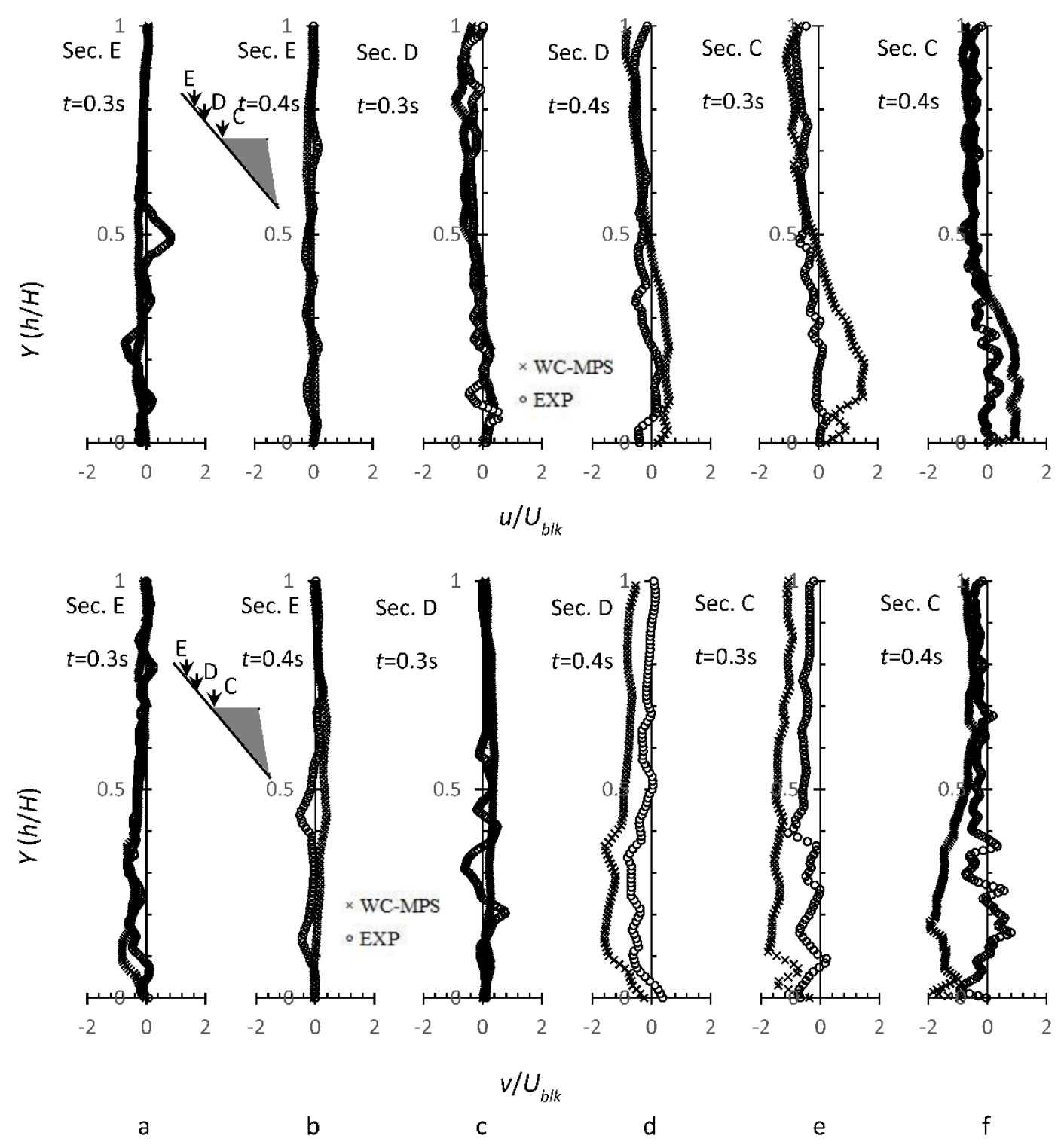

Figure 4-8. The comparison of velocity distribution for the cross-section C, D and E of Case 2 at $t=0.3 \mathrm{~s}$ and $0.4 \mathrm{~s}$ 


\subsection{Section-averaged velocity}

The numerical simulated section-averaged velocity distribution for case I and case II are illustrated in Figure 4-9. The horizontal-axis is normalized by dividing the location of selected section $(X)$ to the length of the top edge of the sliding block $(L)$, i.e. the $X / L=0$ is at the left corner of the block, and $X / L=1$ is at the right top corner of the block. Both cases show a relatively similar velocity distribution at each time step. The water velocity distribution for both cases are relatively flat at $t=0.10 \mathrm{~s}$ and subsequently increases with the movement of the sliding block in the range $X / L \in[0.0,0.6]$ at $t=0.20 \mathrm{~s}$. At $t=0.30 \mathrm{~s}$, and 0.40 s, it can be observed that the higher velocity is distributed in the range $X / L \in[0.0,0.5]$, which represents the major affected area due to the block freely sliding down the plane on the top region. According to the velocity distribution, the relatively higher velocity distributed in the range $X / L \in[1,1.5]$ occurs at the front of the sliding block. In the range of $X / L \in[2.5,3.5]$, the velocity distribution is relatively constant suggesting that the block will not affect the water flow beyond that range. 


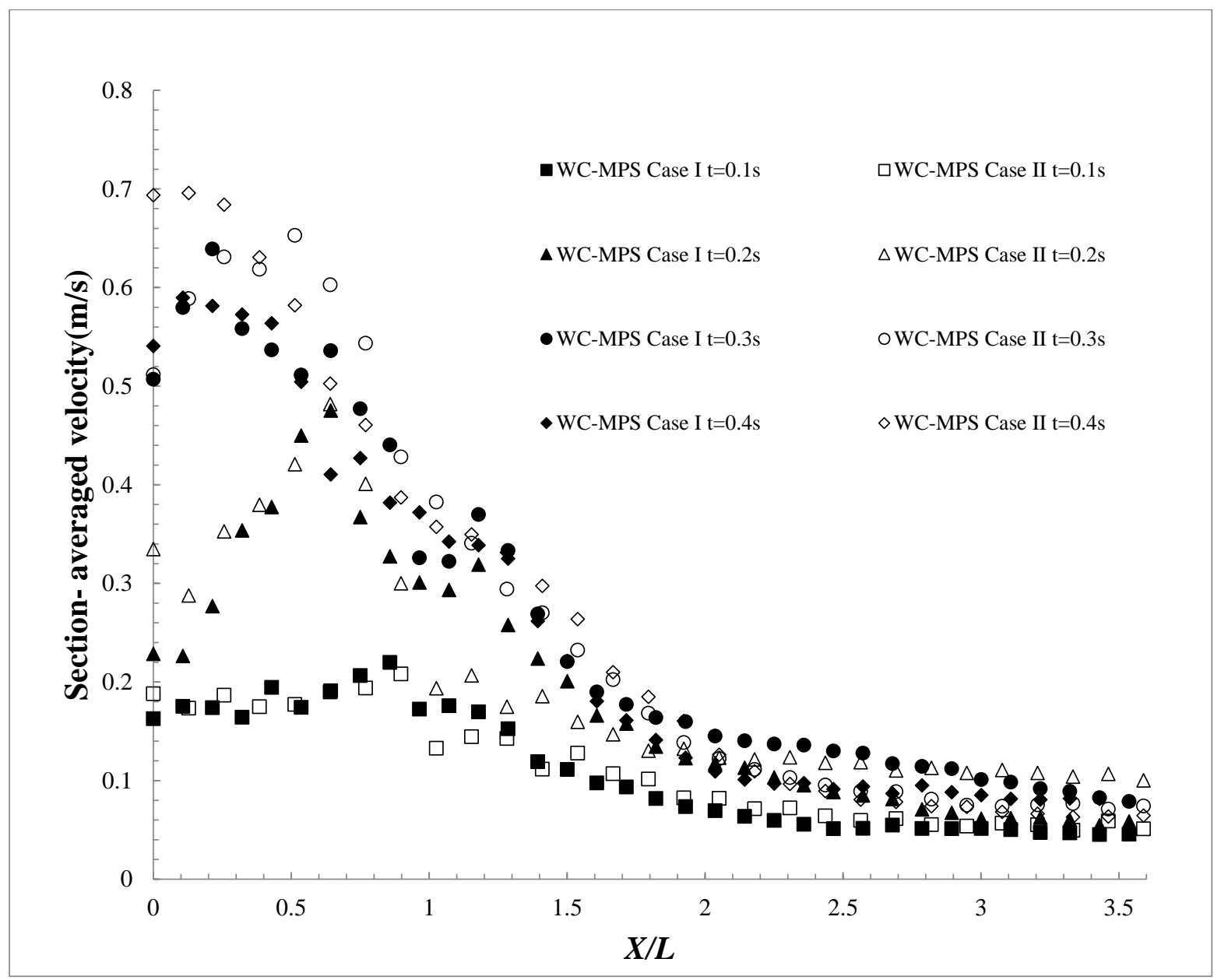

Figure 4-9. Section-averaged velocity distribution of Case I and Case II 


\subsection{Velocity contour and Vorticity}

In Figure 4-10, $U=\sqrt{u^{2}+v^{2}}$ is the calculated speed for case I and case II. The velocity contours illustrate that the water flow has been disturbed around the sliding block with respect to the higher velocity distribution at $t=0.10 \mathrm{~s}$ and $0.20 \mathrm{~s}$ for both cases. As shown in Figure 4-10, the region between the water surface to the top edge of the block has been defined as top area; the region in front of the block has been defined as the front area and the region on the shore has been defined as back area. Similar to the findings from the detailed analysis of the cross-section velocity distribution, water flow in the top area is violently disturbed during the movement of the block down the respective inclination slopes at $t=0.30 \mathrm{~s}$ and $0.40 \mathrm{~s}$. The front of the block in case $\mathrm{I}$ is more violently affected as compared to case II at $t=0.30 \mathrm{~s}$. Consequently, the front area for both cases show relatively constant magnitude at $t=0.40 \mathrm{~s}$. From Figure $4-10$, the back area for both case I and case II show the least influence from the movement of the sliding block. Furthermore, a circulation movement, which is considered as the major influencing factor to water flow, is distinguishable at the top corner for both cases at $t=0.40 \mathrm{~s}$.

Figure 4-11 illustrates the vorticity with the normal velocity vector. The vorticity has been calculated based on the velocity; specifically, the vorticity has been known as $\bar{\omega}$ and calculated as:

$$
\bar{\omega}=\left(\frac{\partial v}{\partial x}-\frac{\partial u}{\partial y}\right)
$$

Recall the Eq.(2.6), the velocity component of horizontal and vertical direction can be expressed by using gradient operator as: 


$$
\begin{aligned}
& \frac{\partial \boldsymbol{v}_{i}}{\partial x}=\frac{d}{n^{0}} \sum_{j \neq i} \frac{\left(\boldsymbol{v}_{j}-\boldsymbol{v}_{i}\right)}{r_{i j}^{2}}\left(y_{j}-y_{i}\right) W\left(r_{i j}, r_{e}\right) \\
& \frac{\partial \boldsymbol{u}_{i}}{\partial y}=\frac{d}{n^{0}} \sum_{j \neq i} \frac{\left(\boldsymbol{u}_{j}-\boldsymbol{u}_{i}\right)}{r_{i j}^{2}}\left(x_{j}-x_{i}\right) W\left(r_{i j}, r_{e}\right)
\end{aligned}
$$

At $t=0.1 \mathrm{~s}$, the vortex can be observed at the right top corner of the block for both cases. Similarly, a relatively large vorticity magnitude is located around the corner while the vortex shows no effect at the top edge of the block. The vortex becomes larger and a clear rotation of the water particles can be observed at $t=0.20 \mathrm{~s}$. A higher intensity vorticity distribution near the right top corner of the block is observed for case I as compared to case II. Moreover, the moving direction of the vortex is from the top corner to the shore. Therefore, the vortex is starting to have an influence at the top edge of the block and shore. It can be observed that the water particles develop to the higher vorticity magnitude in the top area while a negative vorticity distribution occur near the shore in case II. At $t=0.30 \mathrm{~s}$, higher vorticity magnitude is distributed near the center of the top edge of the block with a negative vorticity distributed right above the top edge. The change in vorticity distribution suggests that the rotational direction is transitioning from counterclockwise to clockwise direction in this area. In addition, a second vortex is appearing in both case I and case II at the water surface; however, the second vortex for case II shows a higher vorticity magnitude than case I. Furthermore, the water flow starts to separate in two directions; one is moving along with the block and another is moving to the water surface. A visible separation of the water flow can be seen at $t=0.40 \mathrm{~s}$ and the center location of the vortex relatively remains the same. Additionally, the vorticity distribution near the right top corner of the block is sporadically discontinuous in case I. In case II, vortex 1 and vortex 2 shows 
steady growth and magnitude at $t=0.40 \mathrm{~s}$. Similarly, another phenomenon is observed in that a higher negative vorticity advection near the bed rather than right above the top edge of the block occurs only occurs in case II.

Figure 4-11 illustrates the development of two vortexes from $t=0.10 \mathrm{~s}$ to $t=0.40 \mathrm{~s}$ near the right top corner of the sliding block. The intensity in the development of vortex 1 and 2 increases at each time step. The location in the development of the two vortexes is similar along the sliding block, the magnitude of the vorticity distribution is different between the two cases. According to the development of the first vortex, it can be expected that the second vortex will have the most important influence on the shore. Overall, the vortex will increase in size due to the block's movement and it is the most significant factor that disturbs the water flow. 

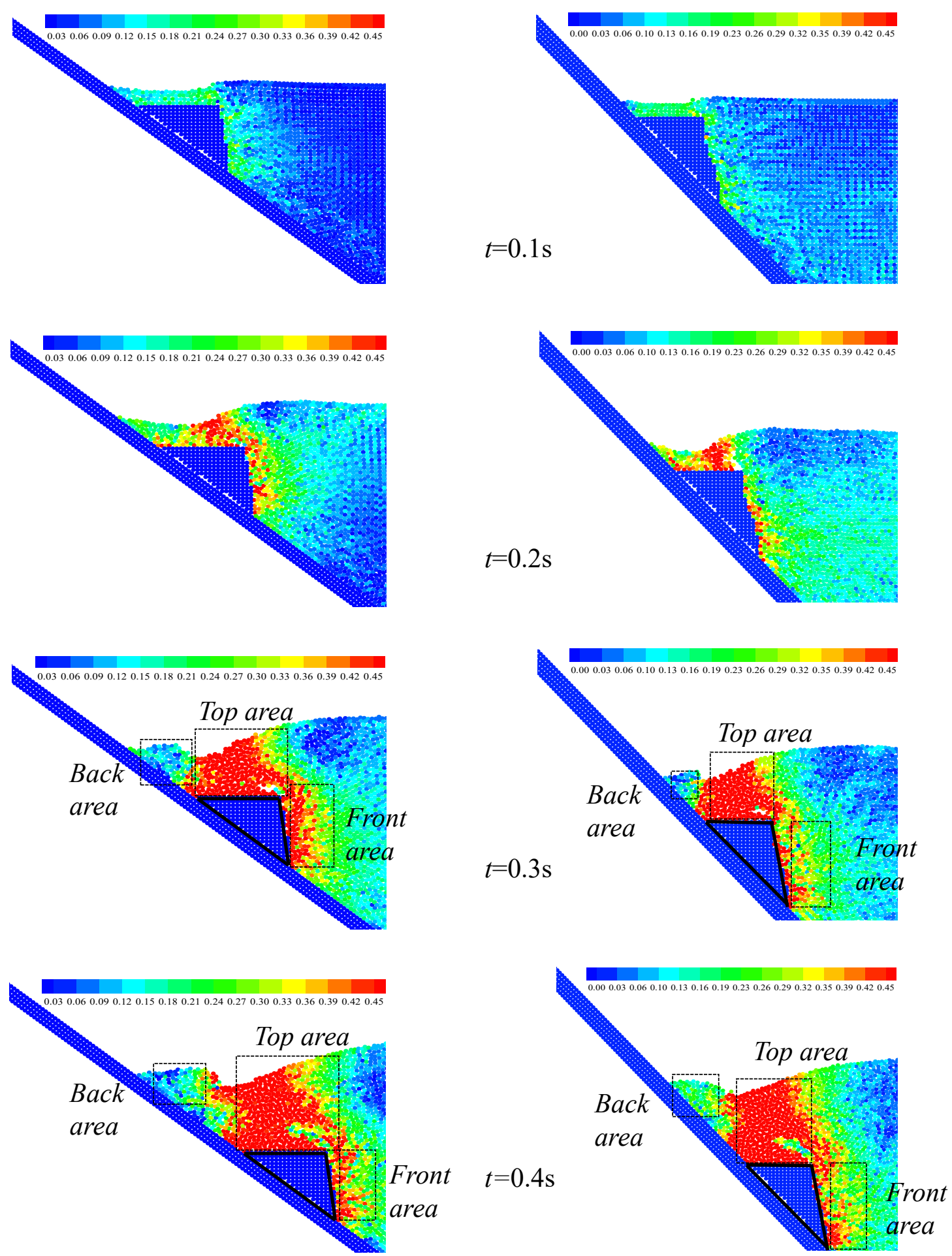

Figure 4-10. Velocity contour of case I (left) and case II (right) 

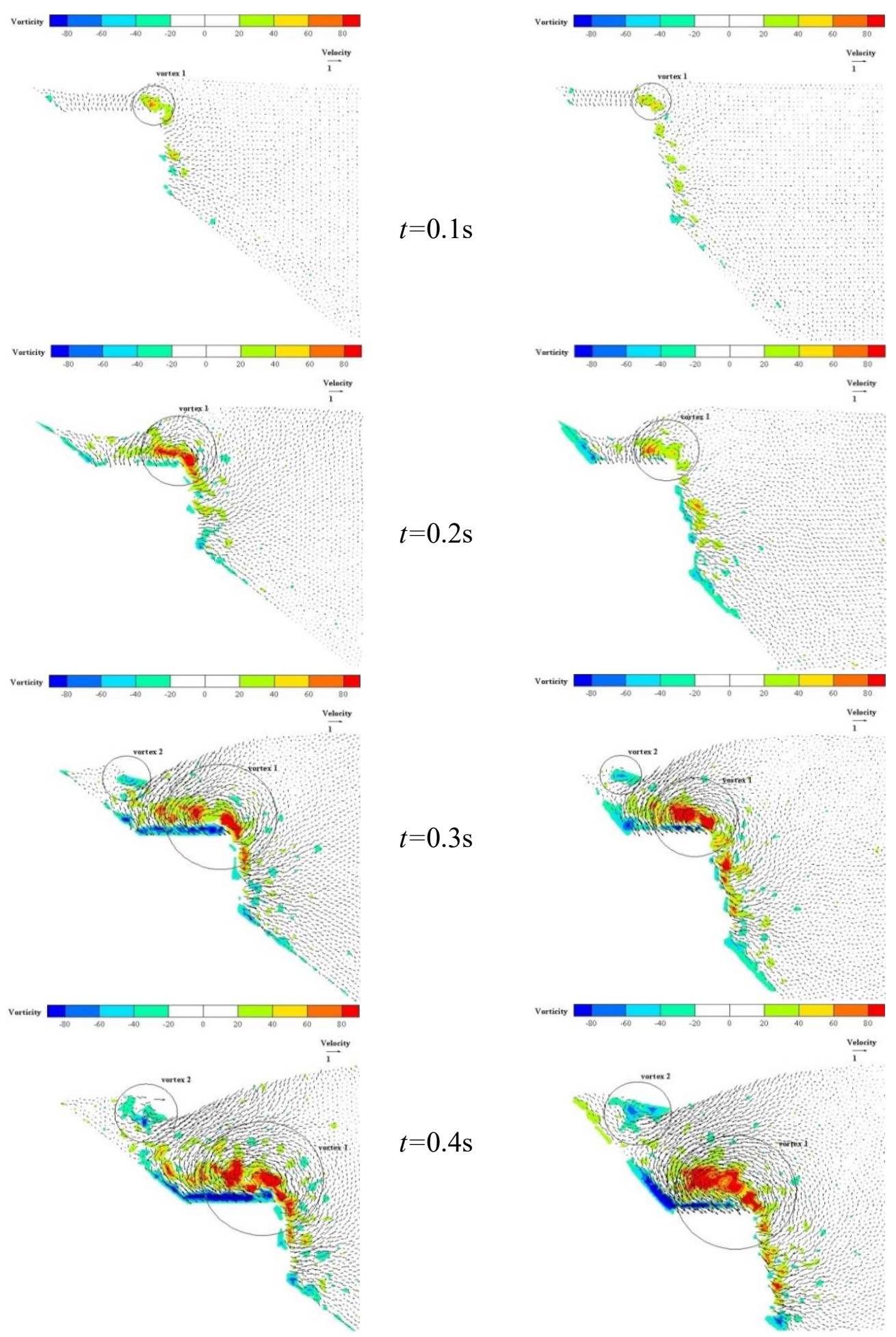

Figure 4-11. Vorticity with the vector plot; left part is showing Case I, and right side are Case II. 


\section{Chapter 5 : SUMMARY}

This study aims at a detailed investigation on the landslide generated impulsive wave and the associated velocity fields with experimental measurement and numerical simulation by the WC-MPS. Two different bed slope angles are investigated in this study. Case I represents an inclination slope of $40^{\circ}$ and case II on a $50^{\circ}$ inclined slope angle. In both cases, a rigid sliding block was employed for wave generation. The top of the sliding block was placed parallel to the water level and $0.02 \mathrm{~m}$ below the water surface. The contributions completed by this study can be summarized as:

- In this study, the inclined slope angles are chosen differed from other researcher. It is important to represent the characteristics of landslides at different bed slope angles in order to evaluate the social and economic impacts from such events. A Validation of the deformational water surface showed good agreement between the experimental and simulated results. The WC-MPS method showed that it has the capacity to accurately simulate landslide tsunamis.

- Comparison of the velocity distribution is completed between the simulation results and experimental data at specific locations along the sliding block. The analysis is unique in that there is limited information in the literature detailing the characteristics in wave generation from inclination slopes. The findings suggest that the velocity distribution will have little effects at specified locations of the sliding block due to different inclination slope angles. Furthermore, this study

presents the difference of numerical simulated results between two different particle sizes. In Appendix, as the Figure A-5 showing, the simulated results are 
showing at two different particle sizes; one is $0.002 \mathrm{~m}$ which generates 431773 particles among the simulation domain, and another one is $0.005 \mathrm{~m}$ which gives 42336 particles as introduced previously. Compare to the experimental data, the simulated results by using smaller particle size is smoothing the trend and the magnitude; however, the significant improvements of using smaller particle size are limited and the computational time is increasing over 7 times.

- The section-averaged velocity plotting displays that the slider will only have the majority influence to the flow filed in a range of two times length of the top surface of the slider. After this range, the flow filed is relatively maintained in a stable condition.

- The velocity and vorticity plots show the development of the flow field distribution and are representative of the movement and vortex development. The vortex has the most significant influence on the water flow caused by the movement of the sliding block with most of the disturbed area located at the top region. However, there was little effect on the velocity distribution on the shore.

In the present study, a satisfactory agreement between the experimental and simulated results using the WC-MPS method of wave generation from a sliding block under two different inclination angles was completed. With the ability of the WC-MPS method replicating actual landslide behaviors, it provides a credible method in evaluating the impacts of wave generated landslides. 


\section{REFERENCES}

Abadie, S., Morichon, D., Grilli, S., Glockner, S., 2010. Numerical simulation of waves generated by landslides using a multiple- fl uid Navier - Stokes model. Coast. Eng. 57, 779-794. https://doi.org/10.1016/j.coastaleng.2010.03.003

Antuono, M., Colagrossi, A., Marrone, S., Molteni, D., 2010. Free-surface flows solved by means of SPH schemes with numerical diffusive terms. Comput. Phys. Commun. 181, 532-549. https://doi.org/10.1016/J.CPC.2009.11.002

Ataie-Ashtiani, B., Nik-Khah, A., 2008. Impulsive waves caused by subaerial landslides. Environ. Fluid Mech. 8, 263-280. https://doi.org/10.1007/s10652-008-9074-7

Ataie-Ashtiani, B., Shobeyri, G., 2008. Numerical simulation of landslide impulsive waves by incompressible smoothed particle hydrodynamics. Int. J. Numer. Methods Fluids 56, 209-232. https://doi.org/10.1002/fld.1526

Batchelor, G.K., 1967. An introduction to fluid dynamics. Cambridge University Press.

Colagrossi, A., Landrini, M., 2003. Numerical simulation of interfacial flows by smoothed particle hydrodynamics. J. Comput. Phys. 191, 448-475. https://doi.org/10.1016/S0021-9991(03)00324-3

Enet, F., Grilli, S.T., 2007. Experimental Study of Tsunami Generation by ThreeDimensional Rigid Underwater Landslides. J. Waterw. Port, Coastal, Ocean Eng. 133, 442-454. https://doi.org/10.1061/(asce)0733-950x(2007)133:6(442) 
Fu, L., Jin, Y.C., 2015. Investigation of non-deformable and deformable landslides using meshfree method. Ocean $\quad$ Eng. 109, 192-206. https://doi.org/10.1016/j.oceaneng.2015.08.051

Fritz, H. M., Hager, W. H., \& Minor, H. E. (2001). Lituya Bay case: rockslide impact and wave run-up. Science of Tsunami Hazards, 29(1), 3-23.

Fritz, H.M., 2002. Landslide Generated Impulse Waves Experimental Results. https://doi.org/10.1142/9789814277426_0109

Gingold,R.A. and Monaghan, J.J., 1977. Smoothed particle hydrodynamics: theory and application to non-spherical stars. R. Astron. Soc. 181, 375-389.

Gotoh, H., Shao, S., Memita, T., 2004. SPH-LES Model for Numerical Investigation of Wave Interaction with Partially Immersed Breakwater. Coast. Eng. J. 46, 39-63. https://doi.org/10.1142/S0578563404000872

Grilli, S.T., Watts, P., 1999. Modeling of waves generated by a moving submerged body. Applications to underwater landslides. Eng. Anal. Bound. Elem. 23, 645-656. https://doi.org/10.1016/S0955-7997(99)00021-1

Grilli, S.T., Watts, P., 2005. Tsunami Generation by Submarine Mass Failure. I: Modeling, Experimental Validation, and Sensitivity Analyses. J. Waterw. Port, Coastal, Ocean Eng. 131, 283-297. https://doi.org/10.1061/(asce)0733-950x(2005)131:6(283) 
Heinrich, P., 1992. Nonlinear water waves generated by submarine and aerial landslides $118,249-266$.

Heller, V., Bruggemann, M., Spinneken, J., Rogers, B.D., 2016. Composite modelling of subaerial landslide-tsunamis in different water body geometries and novel insight into slide and wave kinematics. Coast. Eng. 109, 20-41. https://doi.org/10.1016/J.COASTALENG.2015.12.004

Higman, B., Shugar, D.H., Stark, C.P., Ekström, G., Koppes, M.N., Lynett, P., Dufresne, A., Haeussler, P.J., Geertsema, M., Gulick, S., Mattox, A., Venditti, J.G., Walton, M.A.L., McCall, N., Mckittrick, E., MacInnes, B., Bilderback, E.L., Tang, H., Willis, M.J., Richmond, B., Reece, R.S., Larsen, C., Olson, B., Capra, J., Ayca, A., Bloom, C., Williams, H., Bonno, D., Weiss, R., Keen, A., Skanavis, V., Loso, M., 2018. The 2015 landslide and tsunami in Taan Fiord, Alaska. Sci. Rep. 8, 1-12. https://doi.org/10.1038/s41598-018-30475-w

Jin, Y.C., Guo, K., Tai, Y.C., Lu, C.H., 2016. Laboratory and numerical study of the flow field of subaqueous block sliding on a slope. Ocean Eng. 124, 371-383. https://doi.org/10.1016/j.oceaneng.2016.07.067

Kondo, M., Koshizuka, S., 2011. Improvement of stability in moving particle semi-implicit method. Int. J. Numer. Methods Fluids 65, 638-654. https://doi.org/10.1002/fld.2207 
Koshizuka, S., Oka, Y., 1996. Moving-Particle Semi-Implicit Method for Fragmentation of Incompressible Fluid. Nucl. Sci. Eng. 123, 421-434. https://doi.org/10.13182/nse96-a24205

Koshizuka, S., Nobe, A., Oka, Y., 1998. Numerical analysis of breaking waves using the moving particle semi-implicit method. Int. J. Numer. Methods Fluids 26, 751-769. https://doi.org/10.1002/(SICI)1097-0363(19980415)26:7<751::AIDFLD671>3.0.CO;2-C

Lee, B.H., Park, J.C., Kim, M.H., Hwang, S.C., 2011. Step-by-step improvement of MPS method in simulating violent free-surface motions and impact-loads. Comput. Methods Appl. Mech. Eng. 200, 1113-1125. https://doi.org/10.1016/j.cma.2010.12.001

Liu, P.L.F., Wu, T.R., Raichlen, F., Synolakis, C.E., Borrero, J.C., 2005. Runup and rundown generated by three-dimensional sliding masses. J. Fluid Mech. 536, 107144. https://doi.org/10.1017/S0022112005004799

Lucy, L.B., 1977. A numerical approach to the testing of the fission hypothesis. Astron. 82, 1013-1024. https://doi.org/10.1007/s00769-003-0757-y

Lynett, P., Liu, P.L.-F., 2002. A numerical study of submarine-landslide-generated waves and run-up. Proc. R. Soc. London. Ser. A Math. Phys. Eng. Sci. 458, 2885-2910. https://doi.org/10.1098/rspa.2002.0973 
Molteni, D., Colagrossi, A., 2009. A simple procedure to improve the pressure evaluation in hydrodynamic context using the SPH. Comput. Phys. Commun. 180, 861-872. https://doi.org/10.1016/J.CPC.2008.12.004

Monaghan, J.J., Kos, A., 2000. Scott Russell's wave generator. Phys. Fluids 12, 622-630. https://doi.org/10.1063/1.870269

Müller-Salzburg, L., 1987. The vajont slide. Eng. Geol. 24, 513-523. https://doi.org/10.1016/0013-7952(87)90082-2

Qiu, L. chao, Jin, F., Lin, P. zhi, Liu, Y., Han, Y., 2017. Numerical simulation of submarine landslide tsunamis using particle based methods. J. Hydrodyn. 29, 542-551. https://doi.org/10.1016/S1001-6058(16)60767-9

Shakibaeinia, A., Jin, Y.-C., 2009. A weakly compressible MPS method for modeling of open-boundary free-surface flow. Int. J. Numer. Methods Fluids 63, n/a-n/a. https://doi.org/10.1002/fld.2132

Shakibaeinia, A., Jin, Y.C., 2012. Lagrangian multiphase modeling of sand discharge into still water. Adv. Water Resour. 48, 55-67. https://doi.org/10.1016/j.advwatres.2012.03.027

Shakibaeinia, A., Jin, Y.C., 2012. MPS mesh-free particle method for multiphase flows. Comput. Methods Appl. Mech. Eng. 229-232, 13-26. https://doi.org/10.1016/j.cma.2012.03.013 
Slingerland, R.L., Voight, B., 1979. Occurrences, properties, and predictive models of landslide-generated water waves. Dev. Geotech. Eng. 14, 317-394. https://doi.org/10.1016/B978-0-444-41508-0.50017-X

Tajnesaie, M., Shakibaeinia, A., Hosseini, K., 2018. Meshfree particle numerical modelling of sub-aerial and submerged landslides. Comput. Fluids 172, 109-121. https://doi.org/10.1016/j.compfluid.2018.06.023

Tanaka, M., Masunaga, T., 2010. Stabilization and smoothing of pressure in MPS method by Quasi-Compressibility. J. Comput. Phys. 229, 4279-4290. https://doi.org/10.1016/J.JCP.2010.02.011

Thielicke, W., Stamhuis, E.J., 2014. PIVlab - Towards User-friendly, Affordable and Accurate Digital Particle Image Velocimetry in MATLAB. J. Open Res. Softw. https://doi.org/10.5334/jors.bl

Watts, P., 1997. Water waves generated by underwater landslides.

Wiegel, R.L., 1955. Laboratory Studies of Gravity Waves Generated by the Movement of a Submerged Body. Am. Geophys. Union 36, 759-774.

Willert, C.E., Gharib, M., 1991. Digital particle image velocimetry. Exp. Fluids. https://doi.org/10.1007/BF00190388 
Xu, T., Jin, Y.C., 2016. Improvements for accuracy and stability in a weakly-compressible $\begin{array}{lllll}\text { particle } & \text { method. } & \text { Comput. } & \text { Fluids } & 137,\end{array}$ https://doi.org/10.1016/j.compfluid.2016.07.014

Yeylaghi, S., Moa, B., Buckham, B., Oshkai, P., Vasquez, J., Crawford, C., 2017. ISPH modelling of landslide generated waves for rigid and deformable slides in Newtonian and non-Newtonian reservoir fluids. Adv. Water Resour. 107, 212-232. https://doi.org/10.1016/j.advwatres.2017.06.013

Yim, S.C., Yuk, D., Panizzo, A., Di Risio, M., Liu, P.L.-F., 2008. Numerical Simulations of Wave Generation by a Vertical Plunger Using RANS and SPH Models. J. Waterw. Port, Coastal, Ocean Eng. 134, 143-159. https://doi.org/10.1061/(asce)0733950x(2008)134:3(143)

Yoon, H.Y., Koshizuka, S., Oka, Y., 1999. A particle-gridless hybrid method for incompressible flows. Int. J. Numer. Methods Fluids 30, 407-424. https://doi.org/10.1002/(SICI)1097-0363(19990630)30:4<407::AID-

FLD846>3.0.CO;2-C

Yuk, D., Yim, S.C., Liu, P.L.F., 2006. Numerical modeling of submarine mass-movement generated waves using RANS model. Comput. Geosci. 32, 927-935. https://doi.org/10.1016/j.cageo.2005.10.028 


\section{Appendix:}

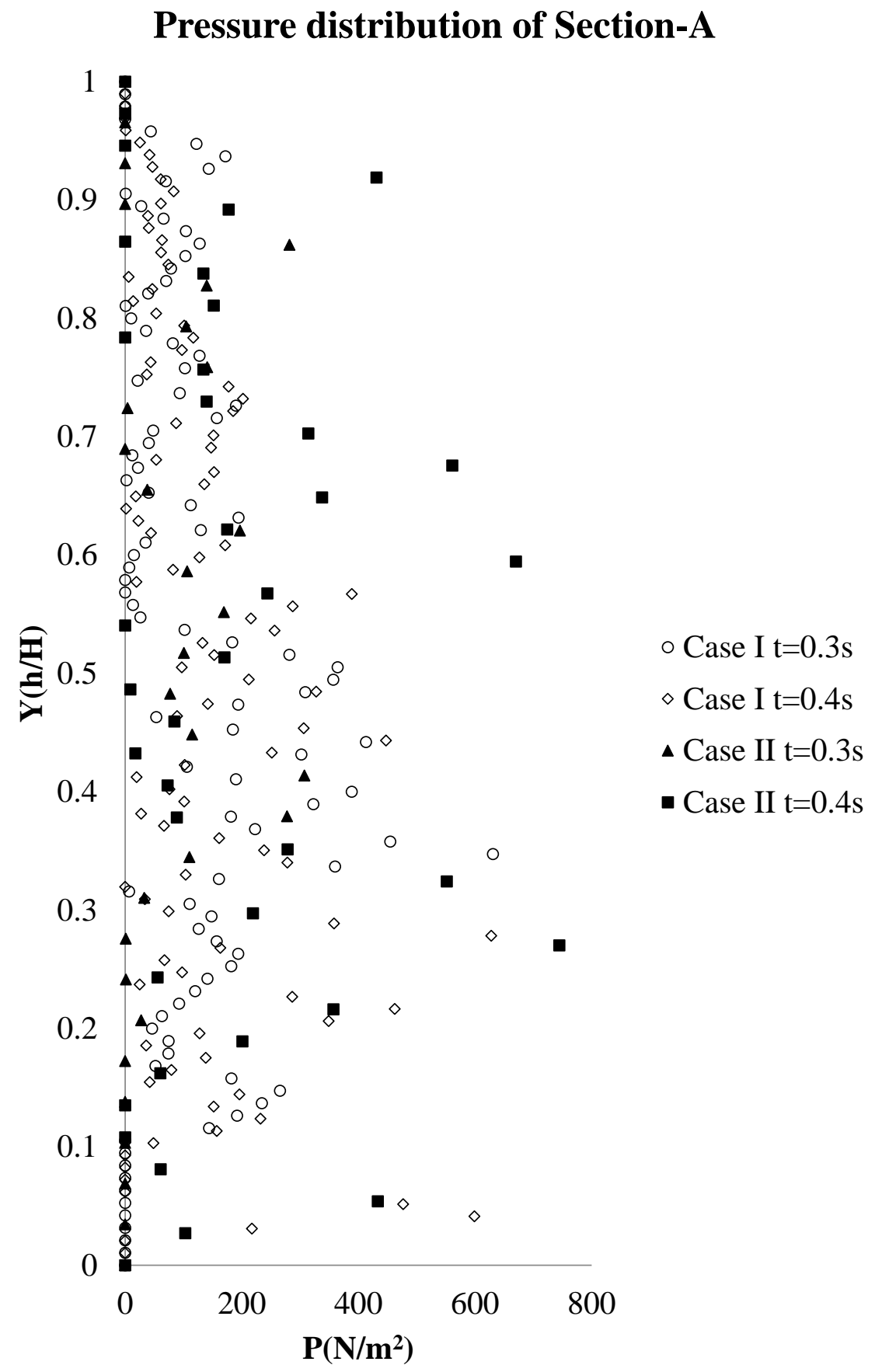

Figure A-1. Pressure distribution at section A. 


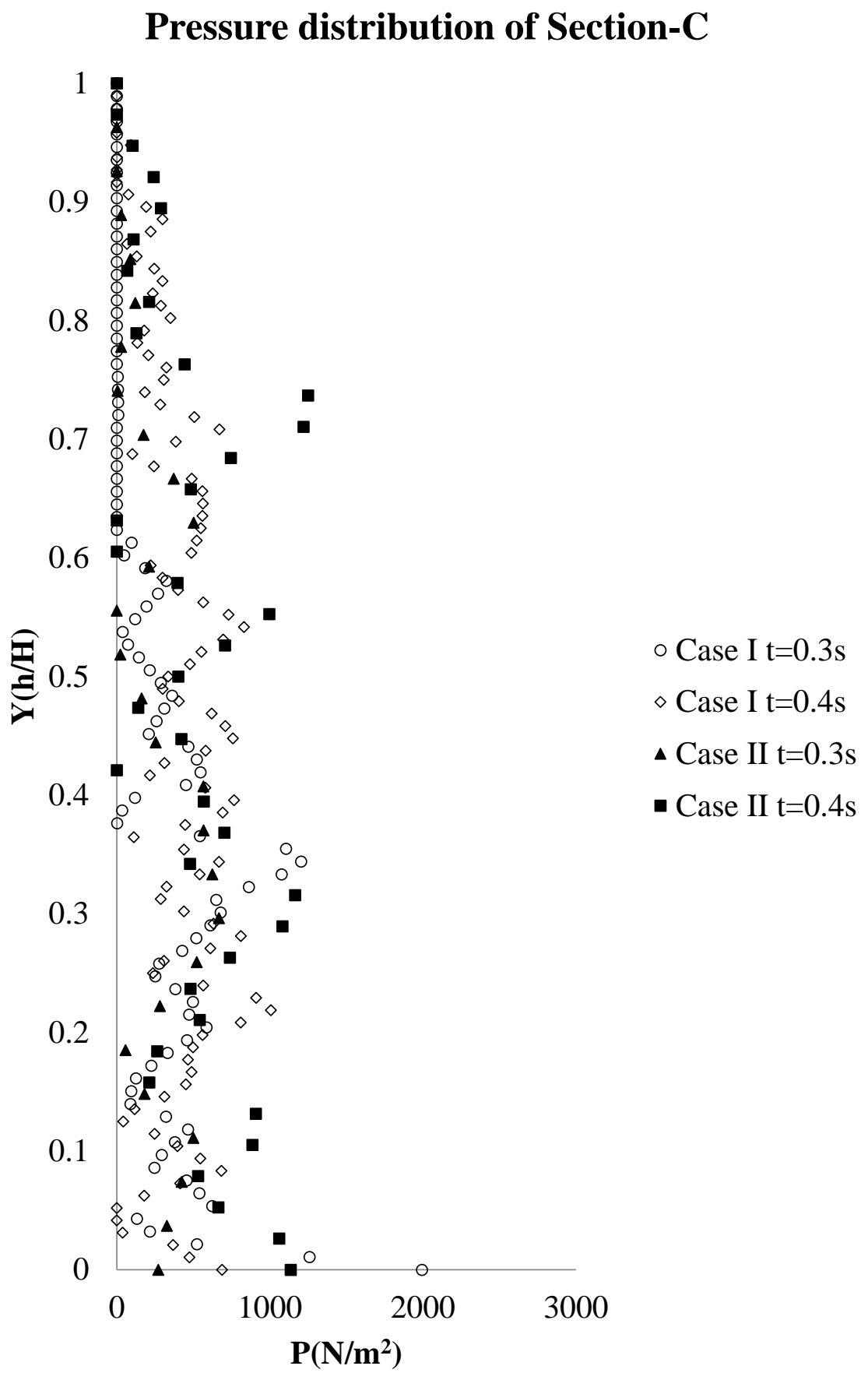

Figure A-2. Pressure distribution at section C. 


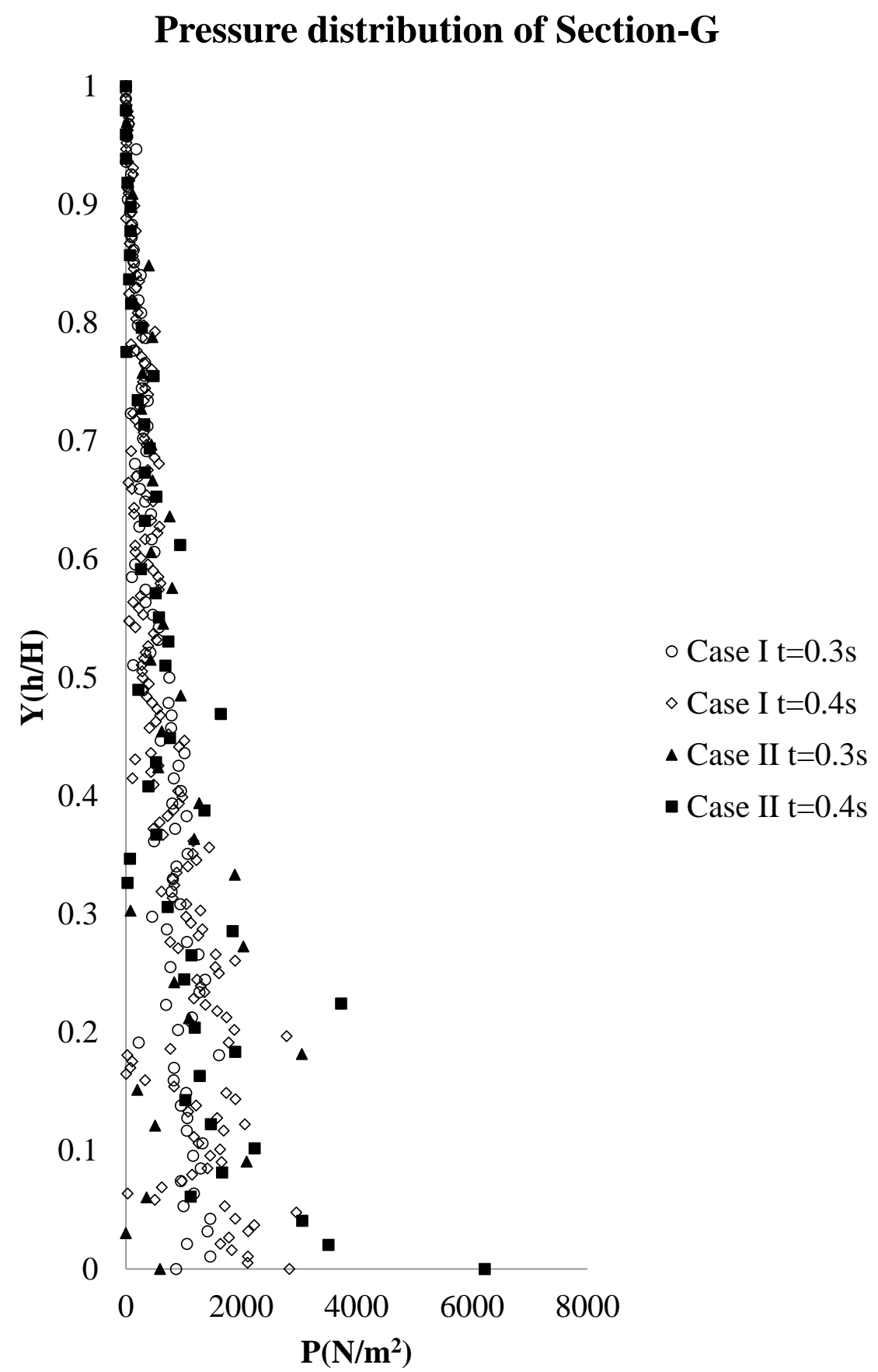

Figure A-3. Pressure distribution at section G. 


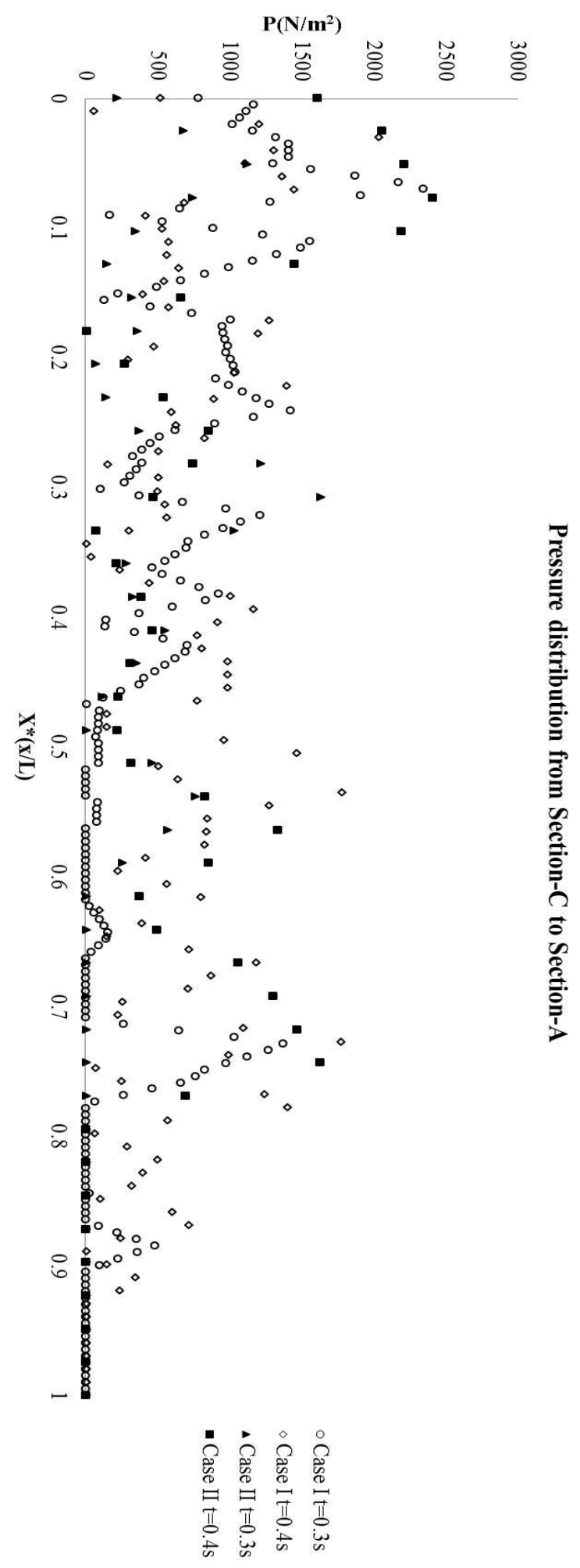

Figure A-4. Pressure distribution from section C to section A horizontally. 

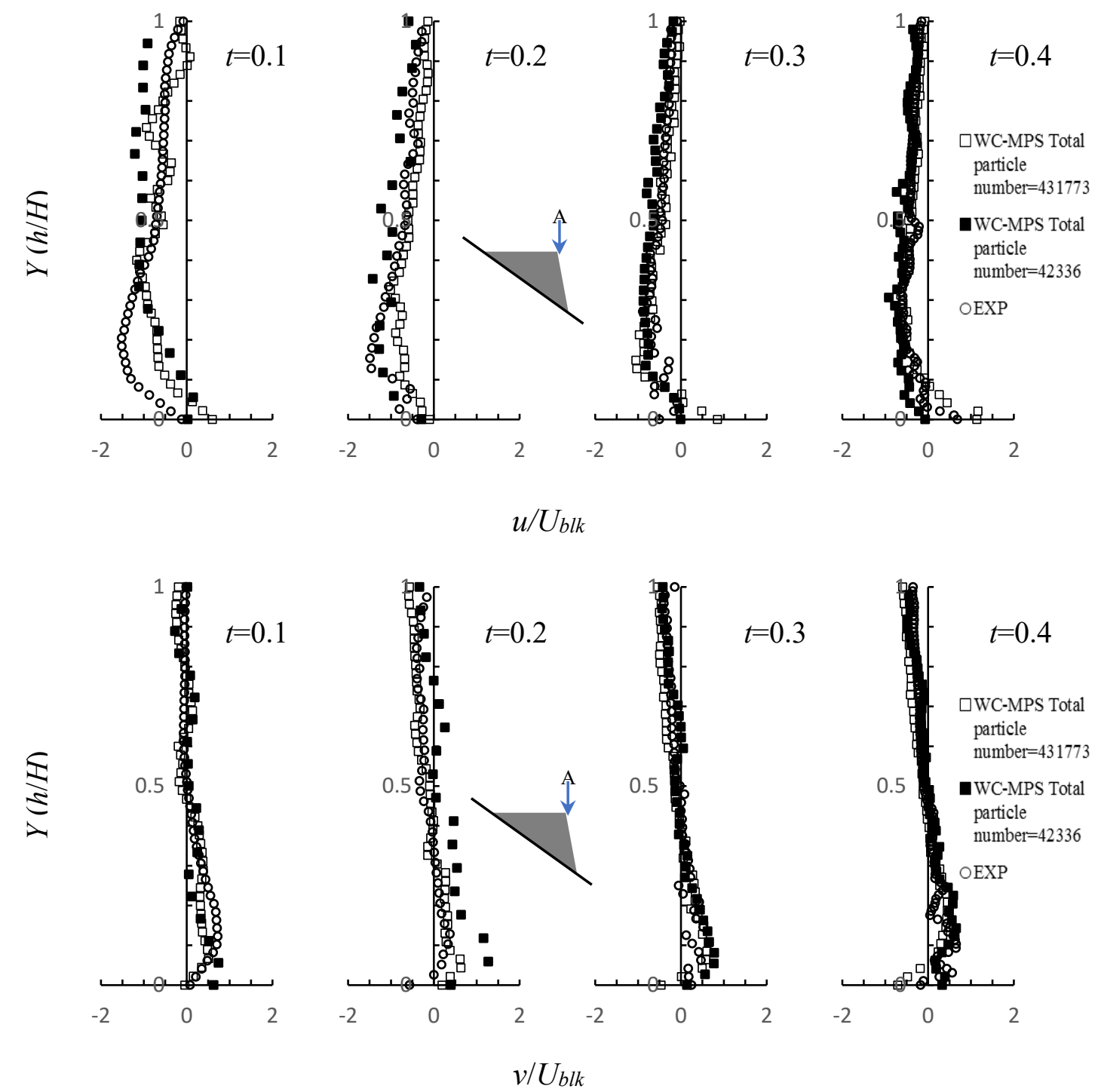

Figure A-5. The comparison of velocity distribution between the experimental data and simulated results with different particle size $(0.002 \mathrm{~m}$ and $0.005 \mathrm{~m})$ at section A in Case I 